\title{
Short Rate Dynamics and Regime Shifts*
}

\author{
HaITAO $\mathrm{LI}^{\dagger}$ AND YUEWU XU ${ }^{\dagger}$ \\ †Stephen M. Ross School of Business, University of Michigan, Ann Arbor, MI, USA and \\ ${ }^{\ddagger}$ Graduate School of Business, Fordham University, New York, NY, USA
}

\begin{abstract}
We characterize the dynamics of the US short-term interest rate using a Markov regime-switching model. Using a test developed by Garcia, we show that there are two regimes in the data: In one regime, the short rate behaves like a random walk with low volatility; in another regime, it exhibits strong mean reversion and high volatility. In our model, the sensitivity of interest rate volatility to the level of interest rate is much lower than what is commonly found in the literature. We also show that the findings of nonlinear drift in Ait-Sahalia and Stanton, using nonparametric methods, are consistent with our regime-switching model.
\end{abstract}

\section{INTRODUCTION}

Modeling the dynamics of the short-term interest rate is one of the most important topics in asset pricing studies. The instantaneous risk-free interest rate or the so-called short rate is the state variable that determines the evolution of the yield curve in an important class of term structure models, such as Vasicek (1977) and Cox et al. (1985) (hereafter CIR). Therefore, it is of fundamental importance for pricing fixed-income securities. Many theoretical models for the short rate have been developed in the literature, and as many empirical studies have been conducted using these models. ${ }^{1}$

In the theoretical term structure literature, the short rate is typically modeled as a time-homogeneous diffusion process

$$
d r_{t}=\mu\left(r_{t} ; \theta\right) d t+\sigma\left(r_{t} ; \theta\right) d B_{t}
$$

* We thank Yacine Ait-Sahalia, Warren Bailey, Yongmiao Hong, Jonathan Ingersoll, Robert Jarrow, and seminar participants at Cornell University and the American Finance Association Meeting for helpful comments. We are especially grateful to the editor, Henry Cao, and an associate editor for insightful comments and suggestions. We also thank Yacine Aït-Sahalia for providing the Eurodollar interest rate data. We are responsible for any remaining errors.

1 Other theoretical models include Beaglehole and Tenney (1992), Brennan and Schwartz (1979), Constantinides (1992), Courtadon (1982), Cox et al. (1980), Dothan (1978), Duffie and Kan (1996), Longstaff and Schwartz (1992), Marsh and Rosenfeld (1983) and Merton (1973). Empirical studies on the short rate include Aït-Sahalia (1996a, b), Andersen and Lund (1997), Ang and Bekaert (1998), Brenner et al. (1996), Brown and Dybvig (1986), Chan et al. (1992), Chapman and Pearson (1999), Chapman et al. (1999), Conley et al. (1997), Gray (1996), and Stanton (1997), among others.

(C) 2009 The Authors. Journal compilation (C) International Review of Finance Ltd. 2009. Published by Blackwell Publishing Ltd., 9600 Garsington Road, Oxford OX4 2DQ, UK and 350 Main Street, Malden, MA 02148, USA. 
where $\left\{B_{t}, t \geq 0\right\}$ is a standard Brownian motion and $\theta$ represents model parameters. The drift and diffusion functions $\mu(\cdot)$ and $\sigma^{2}(\cdot)$ determine the dynamics of the short rate. Most existing models assume that the interest rate exhibits mean-reversion and that the drift is a linear function of the level of the interest rate. They also assume that the diffusion function takes the form of $\left(\sigma r^{\gamma}\right)^{2}$, where $\gamma$ measures the sensitivity of interest rate volatility to the level of interest rate. With $\gamma=0$ and 0.5 , we obtain the famous Vasicek and CIR models, respectively. Despite many empirical studies using the short rate, the evidence on the functional forms of the drift and diffusion is still not conclusive.

Ait-Sahalia (1996b) and Stanton (1997), using nonparametric methods, overwhelmingly reject almost all existing models for the short rate: They find that the drift term is a nonlinear function of the short rate, contrary to the assumption in most models. Both studies show that for the lower and middle ranges of the interest rate, the drift is almost zero but becomes significantly negative when the interest rate is high. These findings lead to the development of nonlinear term structure models, such as that of Ahn and Gao (1999).

However, the evidence of nonlinear drift has been challenged by Pritsker (1998) and Chapman and Pearson (1999), who find that the nonparametric methods of Ait-Sahalia (1996b) and Stanton (1997) have severe small sample problems, especially near extreme observations. This bias can cause one to find nonlinearity in the drift when it is in fact linear.

Controversies about the diffusion term also exist in the literature. On one hand, there seems to be some consensus that $\gamma$ is somewhere between 1 and 2 . For example, Chan et al. (1992) (hereafter CKLS) show that in a single factor model of the short rate, $\gamma$ roughly equals 1.5 , and all the models with $\gamma \leq 1$ are rejected. (In this paper, we refer to the model in which $\gamma$ is a free parameter as the CKLS model). The findings in Aitt-Sahalia (1996b), Stanton (1997), and Conley et al. (1997) are largely consistent with CKLS's results.

On the other hand, there are questions about the robustness of this result. For example, the $\gamma=1.5$ model dictates that interest rate volatility is very sensitive to the level of interest rate: Increase in the interest rate necessarily leads to increased volatility, while decrease in the interest rate necessarily leads to decreased volatility. However, as pointed out by Brenner et al. (1996) (hereafter BHK), 'in several historical periods (such as 1983-1984), rates were high but stable, and in several periods (such as late 1992 and early 1993), rates were low but volatility was high.' While this phenomenon can hardly be captured by the traditional models, it could be modeled by a GARCH process or a stochastic volatility model. BHK (1996) and Andersen and Lund (1997) show that a model with $\gamma=0.5$ fits the short rate quite well if $\sigma_{t}^{2}$ follows a GARCH $(1,1)$ process or a stochastic volatility model. Campbell et al. (1997) also suggest that some regime changes might be needed to fit the data over a longer sample period, and that 'it may be that a model with $\gamma=0$ or $\gamma=0.5$ is adequate once regime changes are allowed.' Given these considerations, it is reasonable to conclude that there is no strong consensus in the current literature about what is a good model for the short rate. 
While most of the existing studies model the short rate as a time homogeneous diffusion, it is conceivable that its stochastic behavior might change over time due to changes in monetary policy, business cycle and other macroeconomic factors. Therefore, explicitly modeling the time changing behavior of the short rate might reveal important features of the data that cannot be obtained by studying a time homogeneous model. So in this paper, we study the short rate dynamics from the perspective of a Markov regimeswitching model. Specifically, we focus on the following regime-switching model for the short rate:

$$
d r_{t}=\left[\alpha\left(S_{t}\right)+\beta\left(S_{t}\right) r_{t}\right] d t+\sigma\left(S_{t}\right) r_{t}^{\gamma} d B_{t}
$$

where $S_{t}$ is an unobserved state variable, which follows a continuous time Markov chain with a finite number of states. In this model, interest rate exhibits different behaviors in different regimes. If $\gamma$ equals 0 and 0.5 , we obtain the multi-regime Vasicek and CIR models, respectively. If we allow $\gamma$ to be estimated from the data, we obtain the multi-regime CKLS model.

Since the pioneering work of Hamilton (1989), regime-switching models have been widely used to model the time series behavior of many important economic variables. For applications to the short rate data, see, for example, Gray (1996), Bekaert et al. (1997), and Ang and Bekaert (1998). However, three unique features of our research distinguish it from these studies.

First, whereas the justification of a second regime in the data has been primarily based on economic intuition, we formally test this hypothesis using the likelihood ratio test developed by Garcia (1998), which is extended in this paper to cover a two-regime CKLS model. This is probably one of the first applications of Garcia's results to the short rate data. We are only aware of one other paper that conducts this kind of test; namely, Ang and Bekaert (1998) which uses a test developed by Hansen (1992). However, Hansen's method only provides a bound on the distribution, while Garcia's results provide the exact asymptotic distribution. Hansen's test is also computationally more challenging to implement than Garcia's.

Second, unlike most existing regime-switching models of the short rate, which assume that $\gamma$ equals either 0 or 0.5 , we estimate $\gamma$ as a free parameter from the data in order to examine the effects of regime shifts on such an important parameter.

Finally, unlike most existing studies which focus on the sample period from the late 1960s to the early 1990s (a period in which $\gamma=1.5$ works well), we consider the entire post-World War II era (from January 1946 to December 1998). This allows us to examine the robustness of the findings of CKLS (1992).

We perform our analysis using the daily 7-day Eurodollar rate of Aït-Sahalia $(1996 a, b)$ and the monthly 3-month US T-bill rate from CRSP. Our empirical evidence shows that the short rate can be described as a Markov regimeswitching model with two different regimes: In one regime, interest rate exhibits strong mean-reversion and high volatility; in another regime, it 
behaves like a random walk with low volatility. Our results provide supporting evidence of term structure models that explicitly model regime shifts in the spot rate, such as Bansal and Zhou (2002) and Dai et al. (2007).

These findings have important implications for both the drift and diffusion functions, and thus the dynamics of the short rate. First, we confirm the intuition of Campbell et al. (1997): a regime-switching CIR model can fit the short rate data quite well. We find that in the two-regime CKLS model, $\gamma$ is about 0.5 when estimated using Aït-Sahalia's Eurodollar rate from 1973 to 1995 , and $\gamma$ is about 0.79 using the 3-month T-bill rate from 1946 to $1998 .^{2}$

Second, our regime-switching model is more flexible than the traditional models in describing the conditional volatility of the interest rate. Unlike traditional models in which volatility solely depends on the level of the interest rate, our model allows the volatility to be low while the interest rate is high, and the volatility to be high while the interest rate is low. BHK (1996) specifically points out two historical episodes as challenges to the traditional model: 1983-1984 and 1992-1993. Our model clearly identifies the 1983-1984 period as a low volatility regime and the 1992-1993 period as a high volatility regime.

Finally, our regime-switching model provides evidence of nonlinearity in the drift of the short rate. We apply the nonparametric methods of Ait-Sahalia (1996b) and Stanton (1997) to data simulated from our two-regime model. The nonparametric estimates of the drift using simulated data are very similar to those found by Ait-Sahalia and Stanton using actual interest rate data. Therefore, we show that the nonlinear drift documented in the nonparametric literature could be consistent with an underlying process of the short rate, which has a true nonlinear drift.

The rest of this paper is organized as follows. In Section II, we discuss the estimation and testing of Markov switching models for the short rate. In Section III, we present the empirical findings. In Section IV, we re-examine the evidence of nonlinear drift documented in the nonparametric literature. In Section V, we conclude the paper.

\section{ESTIMATION AND TESTING OF REGIME-SWITCHING MODELS}

In this section, we first discuss the maximum likelihood estimation of regimeswitching models, and then introduce the likelihood ratio test of Garcia (1998) for the number of regimes. We also derive the covariance function for a tworegime CKLS model, which serves as an input to Garcia's test.

2 Bliss and Smith (1998) reach similar conclusions by allowing structural breaks in the time period of Federal Reserve Experiment of October 1979 through September 1982. However, their model does not allow regime shifts outside that period. They do not address the nonlinear drift issue either. 


\section{A. Maximum likelihood estimation of regime-switching models}

We assume that the dynamics of the short rate are given in equation (2). Let $R$ be the rate matrix of the continuous time Markov chain that $S_{t}$ follows. A matrix $R$ is a rate matrix if all of its off-diagonal elements are nonnegative and the sum of the elements in every row is zero. With two regimes where $S_{t}$ equals 0 in the first regime and 1 in the second regime, the rate matrix $R$ is

$$
R=\left[\begin{array}{cc}
-\kappa(0) & \kappa(0) \\
\kappa(1) & -\kappa(1)
\end{array}\right] .
$$

The elements of $R$ determine the probability of transition from one state to another. Thus the transition matrix of $S(t)$ in a time interval of $\Delta t$ is $I+R \Delta t+o(\Delta t)$ Moreover, the stationary distribution of a Markov chain with the above matrix is given by $\pi_{\infty}(0)=\frac{\kappa(1)}{\kappa(0)+\kappa(1)}$ and $\pi_{\infty}(1)=\frac{\kappa(0)}{\kappa(0)+\kappa(1)}$, where $\pi_{\infty}(i)$ denotes the longrun probability that $S_{t}$ equals 0 and 1 , respectively.

In the analysis of this paper, we consider the discretized version of the above continuous-time diffusion process. As shown by Stanton (1997), the discretization bias is largely inconsequential for the daily, weekly and monthly sampling frequencies used in this paper.

Consider the two-regime-switching model for the short rate:

$$
\Delta r_{t}=\alpha\left(S_{t}\right)+\beta\left(S_{t}\right) r_{t-1}+\sigma\left(S_{t}\right) r_{t-1}^{\gamma} \varepsilon_{t}
$$

where $S_{t}$ takes two different values, 0 and 1 , and $\varepsilon_{t}$ has a standard normal distribution. We call the regime in which $S_{t}=0$ the first regime, and the regime in which $S_{t}=1$, the second regime. The transition probabilities of the Markov chain are

$$
\begin{aligned}
& P\left(S_{t}=1 \mid S_{t-1}=1\right)=p \\
& P\left(S_{t}=0 \mid S_{t-1}=0\right)=q .
\end{aligned}
$$

As shown by Hamilton $(1989,1994)$ and Gray (1996), the above regimeswitching model can be estimated using the maximum likelihood method. Let $\theta$ be the model parameters to be estimated, and $n$, the total number of observations. Then the log likelihood function equals

$$
\begin{aligned}
L(\theta) & =\sum_{t=1}^{n}\left[\log \left\{f\left(\Delta r_{t} \mid \Phi_{t-1}\right)\right\}\right] \\
& =\sum_{t=1}^{n}\left[\log \left\{\sum_{i=0}^{1} f\left(\Delta r_{t} \mid S_{t}=i, \Phi_{t-1}\right) \operatorname{Pr}\left(S_{t}=i \mid \Phi_{t-1}\right)\right\}\right]
\end{aligned}
$$

where $f\left(\Delta r_{t} \mid S_{t}=i, \Phi_{t-1}\right) \sim N\left(\alpha\left(S_{t}\right)+\beta\left(S_{t}\right) r_{t-1}, \sigma^{2}\left(S_{t}\right) r_{t-1}^{2 \gamma}\right)$, and $\Phi_{t-1}$ represents the information set available at $t-1$, which we assume contains only the past interest rates. The density of $\Delta r_{t}$ given $\Phi_{t-1} f\left(\Delta r_{t} \mid \Phi_{t-1}\right)$ is a weighted average of $f\left(\Delta r_{t} \mid S_{t}=i, \Phi_{t-1}\right)$, the conditional density of $\Delta r_{t}$ given that $\Delta r_{t}$ would be generated from regime $i$ at $t$. The weight is $\operatorname{Pr}\left(S_{t}=i \mid \Phi_{t-1}\right)$, the ex ante probability that the data would be generated from regime $i$ at $t$ given $\Phi_{t-1}$. 
The likelihood function can be constructed recursively from the following procedure. For each time $t$, given the probability that the data is from regime $j$ at $t-1$, and the transition probability, the ex ante probability that the data would be generated from regime $i$ equals

$$
\begin{aligned}
\operatorname{Pr}\left(S_{t}=i \mid \Phi_{t-1}\right) & =\sum_{j=0}^{1} \operatorname{Pr}\left(S_{t}=i \mid S_{t-1}=j, \Phi_{t-1}\right) \operatorname{Pr}\left(S_{t-1}=j \mid \Phi_{t-1}\right) \\
& =\sum_{j=0}^{1} \operatorname{Pr}\left(S_{t}=i \mid S_{t-1}=j\right) \operatorname{Pr}\left(S_{t-1}=j \mid \Phi_{t-1}\right) .
\end{aligned}
$$

Given this ex ante probability, the conditional density at $t$ is

$$
f\left(\Delta r_{t} \mid \Phi_{t-1}\right)=\sum_{i=0}^{1} f\left(\Delta r_{t} \mid S_{t}=i, \Phi_{t-1}\right) \operatorname{Pr}\left(S_{t}=i \mid \Phi_{t-1}\right) .
$$

Finally, the probability that the data is from regime $i$ at time $t$ based on $\Phi$ can be obtained from the following equation using Bayes rule:

$$
\begin{aligned}
\operatorname{Pr}\left(S_{t}=i \mid \Phi_{t}\right) & =\operatorname{Pr}\left(S_{t}=i \mid \Delta r_{t}, \Phi_{t-1}\right) \\
& =\frac{f\left(\Delta r_{t} \mid S_{t}=i, \Phi_{t-1}\right) \operatorname{Pr}\left(S_{t}=i \mid \Phi_{t-1}\right)}{\sum_{i=0}^{1} f\left(\Delta r_{t} \mid S_{t}=i, \Phi_{t-1}\right) \operatorname{Pr}\left(S_{t-1}=i \mid \Phi_{t-1}\right)} .
\end{aligned}
$$

If we start the above procedure with $\operatorname{Pr}\left(S_{0}=0 \mid \Phi_{0}\right)=\frac{1-p}{2-p-q}$ and $\operatorname{Pr}\left(S_{0}=1 \mid \Phi_{0}\right)=$ $\frac{1-q}{2-p-q}$, and repeat it from $t=0$ to $n$, we obtain the log-likelihood function.

\section{B. Likelihood ratio test of number of regimes}

While the estimation of regime-switching models is straightforward, testing the number of regimes in the data is much more complicated. This is because the standard regularity conditions required by the classical asymptotic theory break down in regime-switching models, and the likelihood ratio statistic no longer follows a Chi-square distribution. Specifically, when testing the null of a singleregime model versus the alternative of a two-regime model, under the null hypothesis, some parameters of interest are not identified, and the Fisher information matrix is singular.

Given the above difficulties, most empirical studies in the literature simply do not conduct any formal statistical test. Instead, they justify the importance of the second regime mainly on economic intuition. This practice increases the danger of model misspecification in many empirical applications. Therefore Hamilton (1994) emphasizes that 'one of the most important hypotheses one would want to test for regime-switch models concerns the number of regimes in the data.' Several attempts have been made to address this challenging problem in the literature.

Hansen (1992) considers the likelihood as a function of unknown parameters and uses the empirical process theory to bound the asymptotic distribution of a standardized likelihood ratio test for regime-switching models. However, 
as Garcia (1998) points out, Hansen's method is very difficult to implement in practice because it involves evaluating the likelihood across a grid of different values for the parameters of interest in the model. For each set of values, the constrained likelihood function must be optimized with respect to the nuisance parameters of the model. Since the computational burden on even the simplest models can be heavy if the grid search over the parameter space is extensive, this method does not appear very promising for the more complicated models. Moreover, Hansen's method provides a bound for the likelihood ratio statistic instead of a critical value, which means that the test may be conservative.

Garcia (1998) avoids this computational burden by treating the transition probability parameters as nuisance parameters and setting the null hypothesis solely in terms of the parameters governed by the Markov state variable. After reformulating the problem in this way, Garcia assumes that the distribution theory proposed by Davies (1977, 1987), Andrews and Ploberger (1994) and Hansen (1996) for inference in the presence of nuisance parameters under the null hypothesis is applicable to regime-switching models. Based on this assumption, he derives analytically the asymptotic null distribution of the likelihood ratio test and related covariance functions of various two-regime models. Through Monte Carlo simulation, Garcia shows that the derived asymptotic distribution offers a very good approximation to the empirical distribution.

Given the apparent success achieved by Garcia's test, we apply his results to a two-regime CKLS model. To do so, we derive the input to the asymptotic distribution, the covariance function for a two-regime CKLS model. This is probably one of the first applications of Garcia's results to the short rate data.

It is convenient for our derivation if the model in equation (3) is re-written as

$$
r_{t}=\alpha\left(S_{t}\right)+\left[\beta\left(S_{t}\right)+1\right] r_{t-1}+\sigma\left(S_{t}\right) r_{t-1}^{\gamma} \varepsilon_{t}
$$

where

$$
\alpha\left(S_{t}\right)=\alpha_{1}+\alpha_{2} S_{t}, \beta\left(S_{t}\right)+1=\beta_{1}+\beta_{2} S_{t}, \text { and } \sigma\left(S_{t}\right)=\sigma_{1}+\sigma_{2} S_{t} .
$$

After making substitutions, we get

$$
r_{t}-\left(\alpha_{1}+\beta_{1} r_{t-1}\right)-\left(\alpha_{2} S_{t}+\beta_{2} r_{t-1} S_{t}\right)=\left(\sigma_{1}+\sigma_{2} S_{t}\right) r_{t-1}^{\gamma} \varepsilon_{t} .
$$

For ease of exposition, let $\xi=\left(\alpha_{1}, \beta_{1}, \sigma_{1}, \gamma\right), \quad \xi=\left(\alpha_{2}, \beta_{2}, \sigma_{2}\right), \quad \eta=(p, q)$, and $\theta=(\xi, \zeta, \eta)$. We assume that the parameters $\vartheta=(\xi, \zeta)$ belong to an open set $\Xi$ with compact support in $R^{7}$. Hence, the whole parameter space for equation (5) is $\Theta=\Xi \otimes[0,1]^{2}$. The conditional density of $r_{t}$ is given by

$p\left(r_{t} \mid S_{t}, \Psi_{t-1}\right)=\frac{1}{\sqrt{2 \pi}\left(\sigma_{1}+\sigma_{2} S_{t}\right) r_{t-1}^{\gamma}} \exp \left\{-\frac{\left[r_{t}-\left(\alpha_{1}+\beta_{1} r_{t-1}\right)-\left(\alpha_{2} S_{t}+\beta_{2} r_{t-1} S_{t}\right)\right]^{2}}{2\left(\sigma_{1}+\sigma_{2} S_{t}\right)^{2} r_{t-1}^{2 \gamma}}\right\}$ 
where $\Psi_{t-1}=\left\{r_{t-1}, \ldots, r_{1}, s_{t-1}, \ldots, s_{1}\right\}$. This allows us to derive the joint likelihood as

$$
p\left(r_{n}, \ldots, r_{1}, s_{n}, \ldots, s_{1} ; \theta\right)=\prod_{t=1}^{n} p\left(r_{t} \mid S_{t}, \Psi_{t-1}\right)
$$

and the marginal likelihood of the observed interest rate as

$$
p\left(r_{n}, \ldots, r_{1} ; \theta\right)=\prod_{t=1}^{n} \sum_{S_{t}=0}^{1} p\left(r_{t} \mid S_{t}, \Psi_{t-1}\right) \operatorname{Pr}\left(S_{t} \mid \Phi_{t-1}\right) .
$$

We are interested in testing the null hypothesis

$$
H_{0} \text { : A single regime CKLS model }
$$

against the alternative

$$
H_{1} \text { : A nondegenerate two - regime CKLS model. }
$$

The likelihood ratio statistic can be defined as

$$
\begin{aligned}
L R T & =\log \frac{\sup _{(\theta) \in H_{1}} p\left(r_{n}, \ldots, r_{1} ; \theta\right)}{\sup _{(\theta) \in H_{0}} p\left(r_{n}, \ldots, r_{1} ; \theta\right)} \\
& =\sup _{\theta \in H_{1}} L(\theta)-\sup _{\theta \in H_{0}} L(\theta)
\end{aligned}
$$

where $L(\theta)$ denotes the log likelihood

$$
L(\theta)=\log p\left(r_{n}, \ldots, r_{1} ; \theta\right) .
$$

Following Garcia, we represent the null as $\zeta=(0,0,0)$. It is important to notice that under $H_{0}, \eta$ is unidentified. Garcia's approach to circumvent this difficulty is to obtain first a stochastic representation of the log likelihood ratio process for fixed $p$ and $q$. This can be done because for fixed $p$ and $q$ in $(0,1)$, the testing problem is regular and can be handled by the classical techniques. Then he maximizes the representation over $p$ and $q$ to obtain the limiting distribution of the likelihood ratio statistic, i.e.

$$
\sup _{\theta \in H_{1}} L(\theta)=\sup _{\eta \in(0,1)^{2}}\left[\sup _{\vartheta \in \Xi} L(\vartheta, \eta)\right] .
$$

We denote the maximum likelihood estimator (hereafter MLE) of $\xi$ under the null hypothesis by $\tilde{\xi}$, and the MLE of $\vartheta$ under the alternative while holding $\eta$ fixed by $\hat{\vartheta}_{\eta}$ :

$$
\tilde{\xi}=\arg \max _{\xi} L\left(\xi, \zeta_{0}, \eta\right), \hat{\vartheta}(\eta)=\arg \max _{\vartheta} L(\vartheta, \eta)
$$

where $\zeta_{0}=(0,0,0)$. We can then represent the likelihood ratio statistic as

$$
L R T=\sup _{\eta \in(0,1)^{2}} \operatorname{LRT}(\eta)
$$


where

$$
\operatorname{LRT}(\eta)=\left[L(\hat{\vartheta}(\eta), \eta)-L\left(\tilde{\xi}, \zeta_{0}, \eta\right)\right]
$$

Before presenting the asymptotic distribution of the likelihood ratio statistic, we introduce the following definitions. The (normalized) score function for fixed $\eta$ is

$$
Z_{\vartheta}(\vartheta, \eta)=n^{-1} \frac{\partial}{\partial \vartheta} L(\vartheta, \eta)
$$

the limit of the covariance matrix of the score functions is

$$
\Lambda\left(\eta, \eta^{\prime}\right)=\lim _{n \rightarrow \infty} n E\left[Z_{\vartheta}(\vartheta, \eta) Z_{\vartheta}\left(\vartheta, \eta^{\prime}\right)^{T}\right]
$$

and the Fisher information matrix for fixed $\eta$ is $I(\eta)=[\Lambda(\eta, \eta)]^{-1}$.

Theorem 1. The likelihood ratio statistic is distributed asymptotically as

$$
L R T \sim \frac{1}{2} \sup _{\eta \in(0,1)^{2}}[G(\eta)]^{2}
$$

where $G(\eta)$ is a Gaussian process with a covariance matrix $K($, ). This kernel is given by $K\left(\eta, \eta^{\prime}\right)=\Gamma_{\eta}^{T} \Lambda\left(\eta, \eta^{\prime}\right) \Gamma_{\eta^{\prime}}$, where $\Gamma_{\eta}^{T}=I_{22}^{-1 / 2}\left(I_{21}, I_{22}\right)$, and $I_{12}, I_{22}$, and $I_{22}$ are the submatrices (partitioned according to $\vartheta=(\xi, \zeta)$ ) of the Fisher information matrix $I$ :

$$
I(\eta)=\left(\begin{array}{ll}
I_{11} & I_{12} \\
I_{21} & I_{22}
\end{array}\right)=\Lambda(\eta, \eta)^{-1}=\left(\begin{array}{ll}
\Lambda_{11} & \Lambda_{12} \\
\Lambda_{21} & \Lambda_{22}
\end{array}\right)^{-1}
$$

Proof. See Garcia (1998) and the reference therein.

The above theorem shows that the likelihood ratio statistic is distributed asymptotically as the supremum of a functional of a Gaussian process with a certain covariance structure. This result provides a criterion for testing the existence of a second regime in Markov switching models.

To implement this test in practice, the critical values must first be obtained by simulating the Gaussian process with the particular covariance kernel. In what follows, we explicitly calculate the elements of $\Lambda\left(\eta, \eta^{\prime}\right)$ for a two-regime CKLS model. First, we derive a representation of the score function.

Lemma 1. The scores evaluated at $\theta_{0}=\left(\xi_{0}, \zeta_{0}, \eta\right)$ for a particular given $\eta \in(0,1) \otimes(0,1)$ of the nuisance parameters are given by: 


$$
\begin{aligned}
& \left.Z_{\alpha_{1}}\right|_{\theta_{0}}=n^{-1} \sum_{t=1}^{n} \frac{\varepsilon_{t}}{\sigma_{0} r_{t-1}^{\gamma}},\left.\quad Z_{\beta_{1}}\right|_{\theta_{0}}=n^{-1} \sum_{t=1}^{n} \frac{\varepsilon_{t}}{\sigma_{0} r_{t-1}^{\gamma-1}}, \\
& \left.Z_{\sigma_{1}}\right|_{\theta_{0}}=n^{-1} \sum_{t=1}^{n} \frac{1}{\sigma_{0}}\left[\varepsilon_{t}^{2}-1\right],\left.\quad Z_{\gamma}\right|_{\theta_{0}}=n^{-1} \sum_{t=1}^{n} \ln r_{t-1}\left[\varepsilon_{t}^{2}-1\right], \\
& \left.Z_{\alpha_{2}}\right|_{\theta_{0}}=n^{-1} \sum_{t=1}^{n} \sum_{S_{t}} \frac{\varepsilon_{t} S_{t}}{\sigma_{0} r_{t-1}^{\gamma}} p_{t},\left.\quad Z_{\beta_{2}}\right|_{\theta_{0}}=n^{-1} \sum_{t=1}^{n} \sum_{S_{t}} \frac{\varepsilon_{t} S_{t}}{\sigma_{0} r_{t-1}^{\gamma-1}} p_{t}, \\
& \left.Z_{\sigma_{2}}\right|_{\theta_{0}}=n^{-1} \sum_{t=1}^{n} \sum_{S_{t}} \frac{S_{t}}{\sigma_{0}}\left[\varepsilon_{t}^{2}-1\right] p_{t}
\end{aligned}
$$

where $\xi_{0}=\left(\alpha_{0}, \beta_{0}, \sigma_{0}, \gamma_{0}\right), \zeta_{0}=(0,0,0)$, and $p_{t}=\operatorname{Pr}\left(S_{t}=s_{t} \mid r_{n} \ldots, r_{1} ; \vartheta, \eta\right)$.

Proof. See Appendix A.

The next lemma provides an explicit evaluation of the covariance kernel using the scores derived in Lemma 1.

Lemma 2. For any $\eta=(p, q)$ and $\eta^{\prime}=\left(p^{\prime}, q^{\prime}\right)$, the matrix $\Lambda\left(\eta, \eta^{\prime}\right)$ is given by

$$
\begin{aligned}
& \Lambda\left(\eta, \eta^{\prime}\right) \\
& =\left(\begin{array}{ccccccc}
\frac{1}{\sigma_{0}^{2}} E r_{1}^{-2 \gamma} & \frac{1}{\sigma_{0}^{2}} E r_{1}^{-2 \gamma+1} & 0 & 0 & \frac{\pi^{\prime}}{\sigma_{0}^{2}} E r_{1}^{-2 \gamma} & \frac{\pi^{\prime}}{\sigma_{0}^{2}} E r_{1}^{-2 \gamma+1} & 0 \\
\frac{1}{\sigma_{0}^{2}} E r_{1}^{-2 \gamma+1} & \frac{1}{\sigma_{0}^{2}} E r_{1}^{-2 \gamma-2} & 0 & 0 & \frac{\pi^{\prime}}{\sigma_{0}^{2}} E r_{1}^{-2 \gamma+1} & \frac{\pi^{\prime}}{\sigma_{0}^{2}} E r_{1}^{-2 \gamma} & 0 \\
0 & 0 & \frac{2}{\sigma_{0}^{2}} & \frac{2}{\sigma_{0}^{2}} E \ln r_{1} & 0 & 0 & \frac{2 \pi^{\prime}}{\sigma_{0}^{2}} \\
0 & 0 & \frac{2}{\sigma_{0}^{2}} E \ln r_{1} & \frac{2}{\sigma_{0}^{2}} E\left[\ln r_{1}\right]^{2} & 0 & 0 & \frac{2 \pi^{\prime}}{\sigma_{0}^{2}} E \ln r_{1} \\
\frac{\pi}{\sigma_{0}^{2}} E r_{1}^{-2 \gamma} & \frac{\pi}{\sigma_{0}^{2}} E r_{1}^{-2 \gamma+1} & 0 & 0 & \frac{\lambda}{\sigma_{0}^{2}} E r_{1}^{-2 \gamma} & \frac{\lambda}{\sigma_{0}^{2}} E r_{1}^{-2 \gamma+1} & 0 \\
\frac{\pi}{\sigma_{0}^{2}} E r_{1}^{-2 \gamma+1} & \frac{\pi}{\sigma_{0}^{2}} E r_{1}^{-2 \gamma} & 0 & 0 & \frac{\lambda}{\sigma_{0}^{2}} E r_{1}^{-2 \gamma+1} & \frac{\lambda}{\sigma_{0}^{2}} E r_{1}^{-2 \gamma+2} & 0 \\
0 & 0 & \frac{2 \pi}{\sigma_{0}^{2}} & \frac{2 \pi}{\sigma_{0}^{2}} E \ln r_{1} & 0 & 0 & \frac{2 \lambda}{\sigma_{0}^{2}}
\end{array}\right)
\end{aligned}
$$

where $\lambda=\min \left(\pi, \pi^{\prime}\right), \pi=\frac{1-q}{2-p-q}$, and $\pi^{\prime}=\frac{1-q^{\prime}}{2-p^{\prime}-q^{\prime}}{ }^{3}$

\section{Proof. See Appendix A.}

This covariance matrix can be used to simulate the Gaussian process in order to obtain the critical values of the asymptotic distribution. A general procedure for simulating the Gaussian process is given by Garcia (1998).

3 It is interesting to note that if we rearrange the parameter vector as $\left(\alpha_{1}, \alpha_{2}, \beta_{1}, \beta_{2}, \sigma_{1}, \sigma_{2}, \gamma\right)$, the matrix becomes a diagonal block matrix. 


\section{EMPIRICAL RESULTS}

In this section, we estimate and test Markov regime-switching models for the US short rate data. The maximum likelihood estimates of model parameters are obtained by minimizing the negative of the log-likelihood function that was constructed in the previous section using numerical optimization. For a detailed description of our numerical optimization algorithm, see Gay (1983).

\section{A. The data}

We use two different data sets in our empirical study. The first is the daily 7-day Eurodollar rate of Aït-Sahalia (1996a,b), with a sample period from June 1, 1973, to February 25, 1995. The second is the monthly 3-month T-bill rate from CRSP with a sample period from January 1946 to December 1998 . We use the first data set for empirical estimation of our models as well as for comparison with previous findings in the nonparametric literature. We use the second data set to check the robustness of our results and to study the behavior of the short rate over a longer historical period. Figure 1 plots the two interest rate series, and Table 1 reports the summary statistics of the two data sets.

\section{B. Regime shifts in the short rate: evidence from eurodollar rate}

In this section, we first estimate the single-regime Vasicek, CIR and CKLS models using the Eurodollar rate. Then we estimate the two-regime versions of
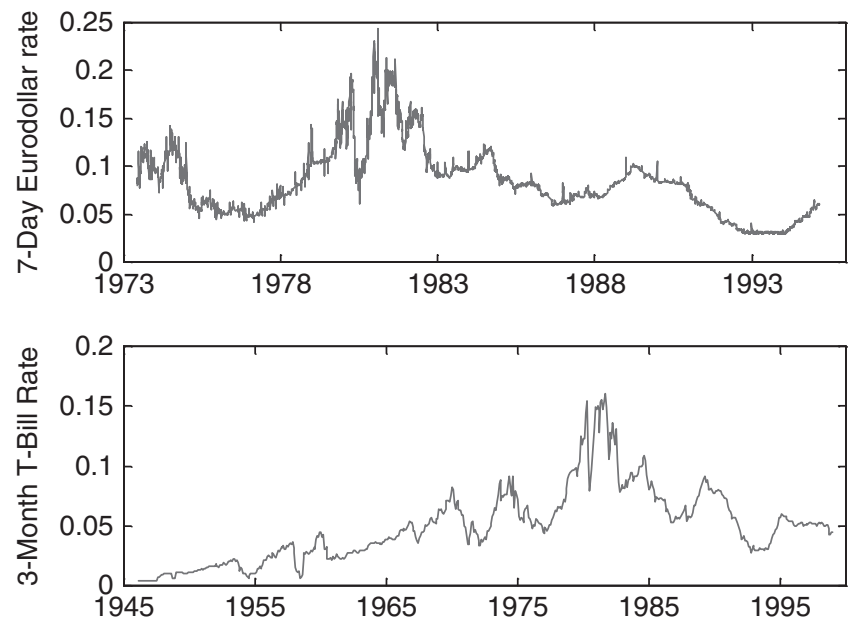

Figure 1 Time Series of Short-Term Interest Rate.

The top panel plots the time series of the daily annualized 7-day Eurodollar rate from June 1, 1973 to February 25, 1995. The bottom panel plots the time series of the monthly 3-month US T-bill rate from January 1946 to December 1998. 
Table 1 Summary statistics

\begin{tabular}{lcccccccr}
\hline \multicolumn{1}{c}{ Mean } & SD & $\rho_{1}$ & $\rho_{2}$ & $\rho_{3}$ & $\rho_{4}$ & $\rho_{5}$ & $\rho_{6}$ \\
\hline Panel A. Bank of America daily & 7-day & Eurodollar rate from & 1973.6 to & 1995.2* \\
$r_{t}$ & 0.08362 & 0.03591 & 0.9389 & 0.8785 & 0.8300 & 0.8014 & 0.7783 & 0.7715 \\
$\Delta r_{t}$ & -0.0000035 & 0.004063 & 0.0214 & -0.0069 & -0.0166 & -0.0024 & 0.0086 & 0.0157 \\
Panel B. CRSP monthly 3-month & T-bill rate from 1946.1 to 1998.12. Total 636 observations ${ }^{\dagger}$ \\
$r_{t}$ & 0.04943 & 0.031 & 0.9849 & 0.9669 & 0.9506 & 0.9350 & 0.9217 & 0.9078 \\
$\Delta r_{t}$ & 0.0064 & 0.0051 & 0.1111 & -0.0669 & -0.0256 & -0.0844 & 0.0234 & -0.1348 \\
\hline
\end{tabular}

*Total 5505 observations. $\rho_{i}$ are monthly autocorrelations of the series at lag $i$. The interest rate is annualized, continuously compounded and expressed in decimal points.

${ }^{\dagger}$ The interest rate is annualized, continuously compounded and expressed in decimal points.

Table 2 Single regime models for eurodollar rate 1973.6-1995.2

\begin{tabular}{lccc}
\hline & Vasicek & CIR & CKLS \\
\hline$\alpha$ & 0.053 & 0.030 & 0.014 \\
$(t$-stat $)$ & $(3.75)$ & $(2.90)$ & $(1.97)$ \\
$\beta$ & -0.0064 & -0.0036 & -0.0012 \\
$(t$-stat $)$ & $(-4.22)$ & $(-2.67)$ & $(-0.95)$ \\
$\sigma$ & 0.41 & 0.12 & 0.02 \\
$(t$-stat $)$ & $(104.35)$ & $(105.34)$ & $(31.91)$ \\
$\gamma$ & 0 & 0.5 & 1.31 \\
$t$-stat $)$ & & & $(87.69)$ \\
Log likelihood & -2843.24 & -1752.55 & -1028.97 \\
\hline
\end{tabular}

Maximum likelihood estimation of single regime models for daily 7-day Eurodollar rate from June 1, 1973, to February 25, 1995. The models being estimated are given by the following equation: $\Delta r_{t}=\alpha+\beta r_{t-1}+\sigma r_{t-1}^{\prime \prime} \varepsilon_{t}$, where $\varepsilon_{t}$ are i.i.d. standard normal. For the Vasicek and CIR models, $\gamma$ equals 0 and 0.5 , respectively, and for the CKLS model, $\gamma$ is estimated from the data. These parameter values correspond to a spot rate that is annualized, continuously compounded, and expressed in percentage terms.

these models. Finally, we provide a formal statistical test for the two-regime CKLS model.

The estimates of the single-regime Vasicek, CIR and CKLS models are, respectively, contained in Columns 2-4 of Table 2. Most of our estimates using the Eurodollar rate are similar to those obtained by CKLS (1992) using the T-bill rate, with the exception of the drift terms. While CKLS (1992) find that the drift parameters of all three models are not statistically significant, we find that they are significant for the Vasicek and CIR models. But we agree with CKLS (1992) in that the speed of mean reversion is not significant for their model. Importantly, we find that $\gamma$ is about 1.3, very close to the value of 1.5 found by CKLS (1992). Our results confirm those of CKLS (1992): There is no strong evidence for mean reversion for the short rate, but $\gamma$ is high and close to 1.5.

The maximum likelihood estimates of the two-regime models using the Eurodollar rate are contained in Table 3. These estimates reveal some interesting 
Table 3 Regime switch models for eurodollar rate

\begin{tabular}{lccc}
\hline & Vasicek & CIR & CKLS \\
\hline$\alpha(0)$ & 0.000051 & -0.000055 & -0.000057 \\
$(t$-stat $)$ & $(0.18)$ & $(-0.16)$ & $(-0.17)$ \\
$\alpha(1)$ & 0.23 & 0.18 & 0.18 \\
$(t$-stat $)$ & $(3.23)$ & $(3.24)$ & $(3.10)$ \\
$\beta(0)$ & -0.000079 & 0.000027 & 0.000030 \\
$(t$-stat $)$ & $(-0.15)$ & $(0.055)$ & $(0.063)$ \\
$\beta(1)$ & -0.020 & -0.017 & -0.017 \\
$(t$-stat $)$ & $(-3.48)$ & $(-3.00)$ & $(-2.91)$ \\
$\sigma(0)$ & 0.075 & 0.030 & 0.029 \\
$(t$-stat $)$ & $(58.02)$ & $(61.60)$ & $(13.29)$ \\
$\sigma(1)$ & 0.83 & 0.26 & 0.26 \\
$(t$-stat $)$ & $(44.05)$ & $(41.29)$ & $(10.84)$ \\
$\gamma$ & 0 & 0.5 & 0.51 \\
$t$-stat $)$ & & & $(13.30)$ \\
$q$ & $(t$-stat $)$ & 0.95 & 0.94 \\
$p$ & $(210.36)$ & $(192.99)$ & $(195.36)$ \\
-stat $)$ & 0.82 & 0.77 & 0.76 \\
Log likelihood & $(53.02)$ & $(40.80)$ & $(39.45)$ \\
\hline
\end{tabular}

Maximum likelihood estimation of two-regime models for daily 7-day Eurodollar rate from June 1, 1973, to February 25, 1995. The model being estimated is given by the following equations: $\Delta r_{t}=\alpha(0)+\beta(0) r_{t-1}+\sigma(0) r_{t-1}^{\prime} \varepsilon_{t}$, if $S_{t}=0 ; \Delta r_{t}=\alpha(1)+\beta(1) r_{t-1}+\sigma(1) r_{t-1}^{\prime} \varepsilon_{t}$ if $S_{t}=1$, where $\varepsilon_{t}$ are i.i.d. standard normal and $S_{t}$ follows a first-order Markov chain whose transition probabilities are given by $p=\operatorname{Pr}\left(S_{t}=1 \mid S_{t-1}=1\right)$ and $q=\operatorname{Pr}\left(S_{t}=0 \mid S_{t-1}=0\right)$. We call the regime in which $S_{t}=0$ the first regime, and the regime in which $S_{t}=1$ is the second. For the Vasicek and CIR models, $\gamma$ equals 0 and 0.5 , respectively, and for the CKLS model, $\gamma$ is estimated from the data. These parameter values correspond to a spot rate that is annualized, continuously compounded, and expressed in percentage terms.

features of the short rate process which are disguised by the single-regime model.

First, we find that the behavior of the short rate is quite different in the two different regimes. In the first regime, the short rate behaves almost like a random walk because all parameters of the drift are close to zero and statistically insignificant. In this regime, $-\alpha(0) / \beta(0)$ ranges from $0.6 \%$ for the Vasicek model to $2 \%$ for the CIR and CKLS models. Since all the data in our sample are above $2 \%$, the drift in the first regime is always positive. ${ }^{4}$ In the second regime, the interest rate has a higher long-run mean, with $-\alpha(1) / \beta(1)$ ranging from $10.8 \%$ to $11.5 \%$ for the three models. It also exhibits strong mean reversion as $\beta(1)$ is negative and statistically significant. The interest rate also has a higher volatility in the second regime, almost eight to 10 times greater than its volatility in the first regime. Our results paint an interesting picture of the dynamics of the short rate: When the interest rate is low, it typically behaves like a random walk with

4 These interpretations have to be taken with caution, given that all the parameters of the drift in the first regime are not statistically significant. 
Table 4 Critical values for the asymptotic distribution of the likelihood ratio statistic

\begin{tabular}{lccccccc}
\hline \% of Distribution & 99 & 95 & 90 & 80 & 70 & 50 & 10 \\
\hline Critical values & 22.36 & 18.01 & 15.81 & 13.51 & 12.06 & 9.78 & 5.43 \\
\hline
\end{tabular}

The critical values are simulated from the asymptotic distribution given in Theorem 1. $\pi$ is chosen from $(0.01,0.99)$ with 0.01 increment, and we simulate the distribution 10,000 times.

low volatility, and when the rate is high, both the speed of mean-reversion and volatility increase. The persistence of the low volatility regime is higher than that of the high volatility regime, given that $q$ equals 0.94 while $p$ ranges from $75 \%$ to $82 \%$ for the three different models.

Second, unlike what has been found in the single-regime model, our estimate of $\gamma$ for the two-regime CKLS model is very close to 0.5 . This suggests that once the effects of regime switching are taken into account, the sensitivity of interest rate volatility to the level of interest rate is greatly reduced, and that a tworegime CIR model provides a good description of the data. This confirms the intuition of Campbell et al. (1997), who argue that in a regime-switching model, 'if regimes with high mean parameters are also with high volatility parameters, then such a model may also explain the apparent sensitivity of interest rate volatility to the level of interest rate without invoking a high value of $\gamma^{\prime \prime}$.

We apply the likelihood ratio statistic developed in the previous section to test the statistical significance of the second regime in the data. Since $\eta$ and $\eta^{\prime}$ enter the covariance matrix $\Lambda\left(\eta, \eta^{\prime}\right)$ only through the function $\pi=\frac{1-q}{2-p-q}$, it is sufficient to set a grid for $\pi$ that is $\left\{\pi_{1}, \ldots, \pi_{N}\right\}$. Table 4 shows the critical values of the asymptotic distribution obtained from simulating the supremum of the functional of the Gaussian process for $\pi \in(0.01,0.99)$ with an increment of 0.01 . The test overwhelmingly rejects the null hypothesis of a single-regime model in favor of a two-regime model. ${ }^{5}$

Many economic arguments have been given for why the regime-switching model proposed here is a reasonable data generating processes for the short rate. For example, Bekaert et al. (1997) interpret the low volatility regime as the 'desired' regime for the monetary authority, since expected inflation is low and real interest rates are at the long-run levels. In such a regime, the interest rate behaves like a random walk, as the monetary authority smooths the shocks that buffet the monetary and real sectors of the economy. In the higher volatility regime, the economy has evolved such that there is now higher expected inflation or possibly higher real interest rates. Aggregate demand may be surging and real growth may be accelerating. As interest rate rises, future short rates are expected to be higher if the monetary authority is unsuccessful at

5 Numerical simulations show that empirical critical values of the likelihood ratio test are close to the asymptotic critical values. See also Figure 1 of Ang and Bekaert (1998). 
Table 5 Single-regime model for 3-month T-bill rate

\begin{tabular}{lccc}
\hline & Vasicek & CIR & CKLS \\
\hline$\alpha$ & 0.081 & 0.044 & 0.042 \\
$(t$-stat $)$ & $(2.12)$ & $(2.50)$ & $(2.85)$ \\
$\beta \alpha$ & -0.015 & -0.0077 & -0.0069 \\
$(t$-stat $)$ & $(-2.29)$ & $(-1.56)$ & $(-1.42)$ \\
$\sigma \alpha$ & 0.50 & 0.19 & 0.16 \\
$(t$-stat $)$ & $(35.69)$ & $(35.57)$ & $(21.88)$ \\
$\gamma \alpha$ & 0 & 0.5 & 0.62 \\
$(t$-stat $)$ & & & $(23.34)$ \\
Log likelihood & -466.13 & -277.18 & -267.88 \\
\hline
\end{tabular}

Maximum likelihood estimation of single regime models for the monthly 3-month T-Bill rate between January 1946 and December 1998. The models being estimated are given by the following equation: $\Delta r_{t}=\alpha+\beta r_{t-1}+\sigma r_{t-1}^{\gamma} \varepsilon_{t}$, where $\varepsilon_{t}$ are i.i.d. standard normal. For the Vasicek and CIR models, $\gamma$ equals 0 and 0.5 , respectively, and for the CKLS model, $\gamma$ is estimated from the data. These parameter values correspond to a spot rate that is annualized, continuously compounded, and expressed in percentage terms.

bringing inflation down. To curb inflation, the monetary authority must now eschew smoothing interest rates. Interest rates may display greater mean reversion as the monetary authority attempts to reduce inflation from its current level, and they will display greater conditional variance with less smoothing. The macroeconomic shocks hitting asset markets may also have greater variance in these uncertain times. ${ }^{6}$

\section{Regime shifts in the short rate: a longer perspective}

To check the robustness of the results obtained from the Eurodollar rate and to study the behavior of the US short rate over a longer historical period, we apply the analysis for the Eurodollar rates to the 3-month US T-bill rate from CRSP for the sample period between January 1946 and December 1998. Table 5 contains our estimates of the single-regime models. We find that, over the entire postWorld War II period, $\gamma$ is only about 0.62 , a value that is much lower than 1.5 but very close to 0.5 .

Table 6 contains the estimates of the three two-regime models for the short rate using the monthly data of the 3-month T-bill rate from January 1946 to December 1998. These results are consistent with our previous findings using the Eurodollar rate. For example, we find that the behavior of the interest rate in the two different regimes is very similar to that of the Eurodollar rate. The first regime is also characterized by low volatility, which is about a quarter of that of the second regime. Given that both $\alpha(0)$ and $\beta(0)$ are positive, the drift in the first regime is always positive. Therefore, the interest rate behaves like a Brownian motion with weakly positive drift in the first regime. In the second

6 This paragraph borrows heavily from Bekaert et al. (1997). 
Table 6 Regime switching models for 3-month T-bill rate

\begin{tabular}{lccc}
\hline & Vasicek & CIR & CKLS \\
\hline$\alpha(0) f$ & 0.016 & 0.014 & 0.0046 \\
$(t$-stat $)$ & $(0.88)$ & $(1.19)$ & $(0.68)$ \\
$\alpha(1)$ & 0.15 & 0.11 & 0.15 \\
$(t$-stat $)$ & $(0.90)$ & $(1.70)$ & $(3.15)$ \\
$\beta(0)$ & 0.0044 & 0.0051 & 0.007 \\
$(t$-stat $)$ & $(0.96)$ & $(1.18)$ & $(2.21)$ \\
$\beta(1)$ & -0.028 & -0.028 & -0.042 \\
$(t$-stat $)$ & $(-1.41)$ & $(-1.97)$ & $(-2.37)$ \\
$\sigma(0)$ & 0.18 & 0.089 & 0.062 \\
$(t$-stat $)$ & $(15.35)$ & $(6.52)$ & $(11.57)$ \\
$\sigma(1)$ & 0.90 & 0.32 & 0.24 \\
$(t$-stat $)$ & $(14.88)$ & $(9.29)$ & $(12.38)$ \\
$\gamma$ & 0 & 0.5 & 0.76 \\
$t$-stat $)$ & & & $(14.75)$ \\
$q$ & & 0.95 & 0.95 \\
$($-stat $)$ & 0.95 & $(27.24)$ & $(15.65)$ \\
$p$ & $(61.80)$ & 0.87 & 0.85 \\
-stat $)$ & 0.88 & $(16.45)$ & $(15.65)$ \\
Log likelihood & $(21.72)$ & -105.90 & -92.627 \\
\hline
\end{tabular}

Maximum likelihood estimation of two-regime models for the monthly 3-month T-Bill rate from 1946.1-1998.12. The models being estimated are given by the following equations: $\Delta r_{t}=\alpha(0)+\beta(0) r_{t-1}+\sigma(0) r_{t-1}^{\prime} \varepsilon_{t}$, if $S_{t}=0$, and $\Delta r_{t}=\alpha(1)+\beta(1) r_{t-1}+\sigma(1) r_{t-1}^{\prime} \varepsilon_{t}$, if $S_{t}=1$, where $\varepsilon_{t}$ are i.i.d. standard normal and $S_{t}$ follows a first-order Markov chain whose transition probabilities are given by $p=\operatorname{Pr}\left(S_{t}=1 \mid S_{t-1}=1\right)$ and $q=\operatorname{Pr}\left(S_{t}=0 \mid S_{t-1}=0\right)$. We call the regime in which $S_{t}=0$ the first regime, and the regime in which $S_{t}=1$ is the second regime. For the Vasicek and CIR models, $\gamma$ equals 0 and 0.5 , respectively, and for the CKLS model, $\gamma$ is estimated from the data. These parameter values correspond to a spot rate that is annualized, continuously compounded, and expressed in percentage terms.

regime, the interest rate has a strong mean-reversion and a high volatility. The long-run mean of the interest rate estimated using T-bill data in this regime is much lower than that estimated using the Eurodollar rates, ranging from 3.7\% to $5.3 \%$ for the three different models. The difference is probably caused by the low T-bill rates in the early part of the sample.

Most importantly, we find that in the two-regime CKLS model, $\gamma$ is roughly 0.76, substantially lower than the value of 1.5 which was found by CKLS (1992). This confirms the intuition of Campbell et al. (1997) that over a longer sample period, a regime-switching CIR model can fit the data well.

In summary, both the T-bill rate and the Eurodollar rate reveal similar time series behaviors of the short rate. In the two-regime model, we find that $\gamma$ is close to 0.5 , and that the interest rate typically wanders around like a random walk with low volatility; when the interest rate becomes high, volatility increases and mean-reversion becomes strong.

Our regime-switching model provides an alternative to the traditional models in describing the conditional volatility of the interest rate. Although generally a high interest rate has a high volatility, our model allows the data to 

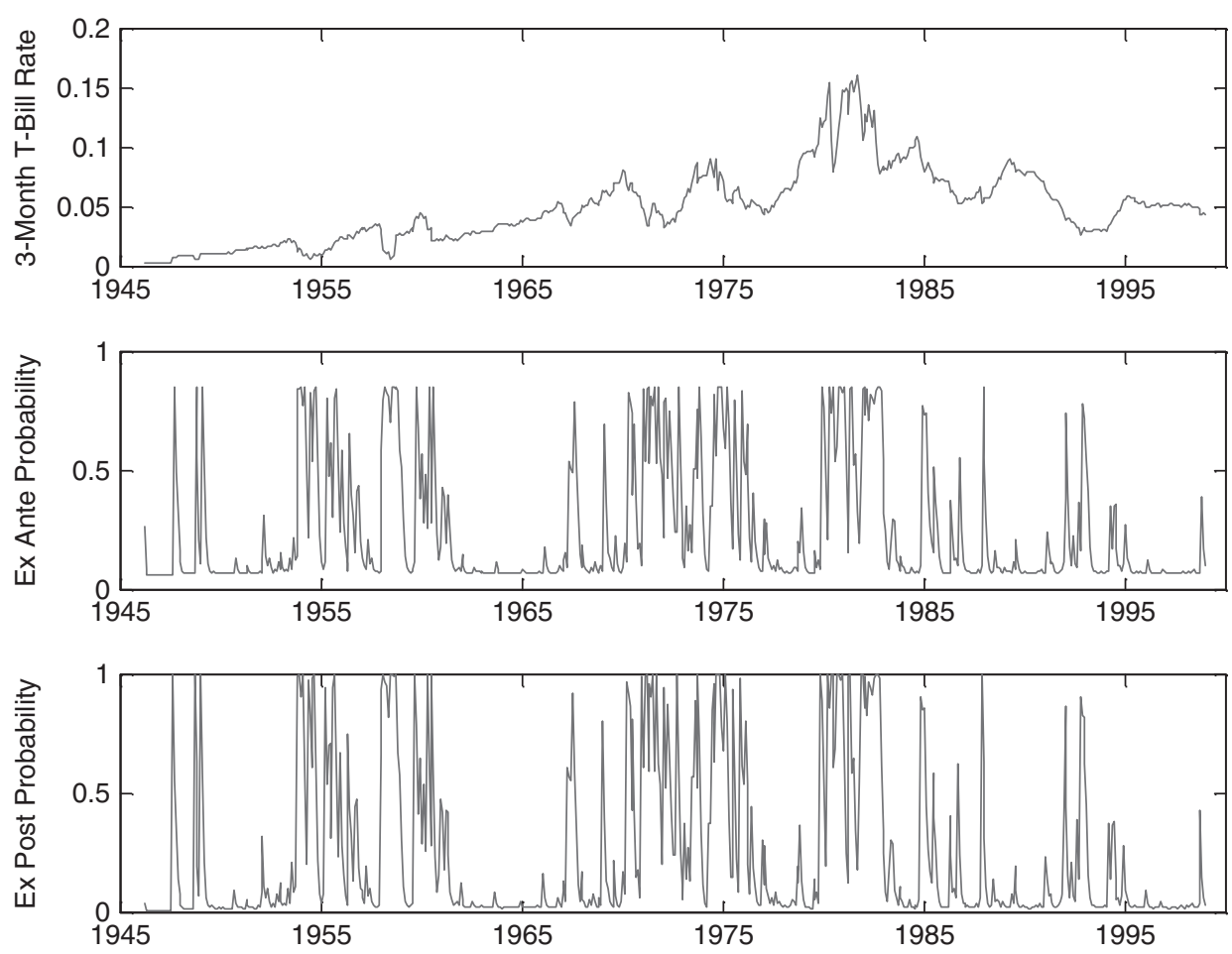

Figure 2 Ex Ante and Ex Post Probability of High Volatility Regime.

The top panel plots the time series of the monthly 3-month US T-bill rate from January 1946 to December 1998. The middle panel plots the ex ante probability that the data is generated from the second regime, i.e., the regime with high volatility and strong meanreversion. The bottom panel plots the ex post probability that the data is generated from the second regime.

be generated from the low volatility regime when the rate is high, and from the high volatility regime when the rate is low. This feature of our model can be seen in Figure 2, which contains the plots of $\operatorname{Pr}\left(S_{t}=i \mid \Phi_{t-1}\right)$ and $\operatorname{Pr}\left(S_{t}=i \mid \Phi_{t}\right)$, respectively, the ex ante and the ex post probability that the world is in regime $i$ at time $t$. We look at the two important episodes in recent history which are specifically pointed out by BHK (1996) as challenges to the traditional models: 1983-1984 and 1992-1993. In the first period, the rate is quite high and grows steadily; in the second period, the rate is low, but volatility is relatively high. Both probability plots clearly identify 1983-1984 as a low volatility regime and 1992-1993 as a high volatility regime. It is hard for the traditional models to match the flexibility of our regime-switching model. ${ }^{7}$

7 Of course, this comparison may not be fair, as our regime switching model is basically a twofactor model. The comparison of the performance of our regime-switching model with the existing two-factor interest rate models would be an interesting topic for future research. 
Table 7 Critical values for the asymptotic distribution of the likelihood ratio statistic

\begin{tabular}{lccccccc}
\hline \% of distribution & 99 & 95 & 90 & 80 & 70 & 50 & 10 \\
\hline Critical values & 22.55 & 19.97 & 15.86 & 13.56 & 12.12 & 9.86 & 5.57 \\
\hline
\end{tabular}

The critical values are simulated from the asymptotic distribution given in Theorem 1. $\pi$ is chosen from $(0.01,0.99)$ with 0.01 increment, and we simulate the distribution 10,000 times.

Following the same simulation procedure as before, we apply the likelihood ratio test to the monthly data. The critical values for the asymptotic distribution are found in Table 7. Again the test overwhelmingly rejects the null hypothesis of a single-regime model.

\section{IS THE SHORT RATE DRIFT NONLINEAR?}

Using nonparametric methods, Aït-Sahalia (1996b) and Stanton (1997) find significant nonlinearity in the drift of the short rate. However, their findings have been challenged by Pritsker (1998) and Chapman and Pearson (1999), who show that the small sample problems in those nonparametric methods could make one find nonlinear drift when the true drift is actually linear. Therefore, the question of whether the drift of the short rate is linear or not has still not been resolved.

Our regime-switching model shows that the drift of the short rate is indeed nonlinear: When the interest rate is low, the short rate typically behaves like a random walk, and when the interest rate is high, the short rate exhibits strong mean-reversion. In this section, we show that the empirical findings of Ait-Sahalia (1996b) and Stanton (1997) are consistent with a true data generating process which follows our regime-switching model. Specifically, we show that when the nonparametric methods are applied to the data simulated from our two-regime model, the estimates of the drift are very similar to those found by Aït-Sahalia (1996b) and Stanton (1997), who use actual US interest rate data.

We simulate data using a two-regime model for the short rate. In each regime, the short rate follows the CIR process:

$$
d r_{t}=\left(\alpha\left(S_{t}\right)+\beta\left(S_{t}\right) r_{t}\right) d t+\sigma\left(S_{t}\right) \sqrt{r_{t}} d B_{t}
$$

Given the initial regime $S_{t-1}$, we simulate $S_{t}$ according to the transition probability of the Markov chain. Then within each regime, given the initial value $r_{t-1}$, we draw successive observations of $r_{t}$ from the transition density of the CIR process, which is noncentral chi-square. 


\section{A. Drift estimated using Aït-Sahalia's method}

We first introduce the estimator used by Aït-Sahalia (1996b), then apply it to the data simulated from our regime-switching model. For the diffusion process given by equation (1), the stationary density of the process equals

$$
\pi(r ; \theta)=\frac{\xi(\theta)}{\sigma^{2}(r ; \theta)} \exp \left\{\int^{r} \frac{2 \mu(u ; \theta)}{\sigma^{2}(u ; \theta)} d u\right\}
$$

where the lower limit of the integration is arbitrary, and $\xi(\theta)$ is a constant that ensures that $\pi(r ; \theta)$ integrates to one. The main idea of Aït-Sahalia's estimation method is that if some parametric forms for $\mu\left(r_{t} ; \theta\right)$ and $\sigma\left(r_{t} ; \theta\right)$ represent the true data generating process, then the stationary density implied by the above equation should be close to that estimated nonparametrically from the data. Aït-Sahalia (1996b) selects the following flexible forms:

$$
\mu\left(r_{t} ; \theta\right)=a_{0}+a_{1} r_{t}+a_{2} r_{t}^{2}+a_{3} r_{t}^{-1}
$$

and

$$
\sigma^{2}\left(r_{t} ; \theta\right)=b_{0}+b_{1} r_{t}+b_{2} r_{t}^{b_{3}}
$$

where $\theta \equiv\left(a_{0}, a_{1}, a_{2}, a_{3}, b_{0}, b_{1}, b_{2}, b_{3}\right)$. Aït-Sahalia $(1996 \mathrm{a}, \mathrm{b})$ proposes a minimum mean square distance measure. In particular, he chooses

$$
\theta^{*} \equiv \arg \min _{\theta \in \Theta} E\left[(\pi(r ; \theta)-\pi(r))^{2}\right]
$$

where the expectation is taken with respect to the true stationary density $\pi(r)$. The estimation is actually performed using

$$
\widehat{\theta}^{*} \equiv \arg \min _{\theta \in \Theta} \frac{1}{n} \sum_{t=1}^{n}\left(\pi\left(r_{t} ; \theta\right)-\widehat{\pi}\left(r_{t}\right)\right)^{2} .
$$

We simulate a two-regime-switching CIR model and apply Aït-Sahalia's procedure to the simulated data. To simplify the task of estimation, we assume that $\sigma_{1}=\sigma_{2}$, and that both are known to the econometrician. Under these assumptions, Chapman and Pearson (1999) show that

$$
\begin{aligned}
\int_{\underline{r}}^{r} \frac{2 \mu(u ; \theta)}{\sigma^{2}(u ; \theta)} d u= & \frac{2 a_{0}}{\sigma^{2}}(\ln r-\ln \underline{r})+\frac{2 a_{1}}{\sigma^{2}}(r-\underline{r}) \\
& +\frac{a_{2}}{\sigma^{2}}\left(r^{2}-\underline{r}^{2}\right)-\frac{2 a_{3}}{\sigma^{2}}\left(\frac{1}{r}-\frac{1}{r}\right) .
\end{aligned}
$$

Therefore, with an estimate of the stationary density, Aït-Sahalia's estimator reduces to a simple ordinary least square problem in a transformation of $\widehat{\pi}\left(z_{i}\right)$, where $z_{i}$ is the equally spaced grid points over the support of the stationary density. Specifically, the dependent variable is $y_{i}=\frac{1}{2} \sigma^{2} \log \left(\widehat{\pi}\left(z_{i}\right) \sigma^{2} z_{i}\right)$, which is regressed on a constant, $\ln z_{i}, z_{i}, \frac{z_{i}^{2}}{2}$, and $-\frac{1}{z_{i}}$. The coefficients of the last four terms are $a_{0}$ through $a_{3}$, respectively.

To match Aït-Sahalia's sample size, we simulate a time series with 5000 observations for 5000 times, and each time we estimate $a_{0}, a_{1}, a_{2}$ and $a_{3}$ using 
Table 8 Parameters used in simulation studies of nonparametric estimators

\begin{tabular}{|c|c|c|c|c|}
\hline & $\alpha\left(S_{i}\right)$ & $\beta\left(S_{i}\right)$ & $\sigma\left(S_{i}\right)$ & $P\left(S_{t} S_{t-1}\right)$ \\
\hline \multicolumn{5}{|c|}{ Panel A. Parameters used in simulation for Ait-Sahalia $(1996 a, b)$ estimator } \\
\hline$S_{t}=0$ & 0.000167 & -0.000027 & 0.02585 & 0.94 \\
\hline$S_{t}=1$ & 0.0018376 & -0.0167 & 0.02585 & 0.76 \\
\hline \multicolumn{5}{|c|}{ Panel B. Parameters used in simulation for Stanton (1997) estimator } \\
\hline$S_{t}=0$ & 0.000000045 & -0.000027 & 0.0003 & 0.94 \\
\hline$S_{t}=1$ & 0.0018376 & -0.0167 & 0.02585 & 0.76 \\
\hline
\end{tabular}

The following parameters are used to simulate a two-regime CIR model which is described by the following continuous time model: $d r_{t}=\left(\alpha(0)+\beta(0) r_{t}\right) d t+\sigma(0) r_{t}^{0.5} d B_{t}$, if $S_{t}=0$, and $d r_{t}=(\alpha(1)+$ $\left.\beta(1) r_{t}\right) d t+\sigma(1) r_{t}^{0.5} d B_{t}$, if $S_{t}=1$, where $B_{t}$ is a standard Brownian motion and $S_{t}$ follows a first-order Markov chain with transition probabilities, $p=\operatorname{Pr}\left(S_{t}=1 \mid S_{t-1}=1\right)$ and $q=\operatorname{Pr}\left(S_{t}=0 \mid S_{t-1}=0\right)$. The simulated data are expressed in percentage terms and converted into decimal numbers before the methods of Stanton and Aït-Sahalia are applied.

Table 9 Estimates of nonlinear drift using Aït-Sahalia's estimator

\begin{tabular}{lcc}
\hline & Estimates from simulated data & Estimates in Aït-Sahalia (1996a, b) \\
\hline$a_{0}$ & -0.002189 & -0.004643 \\
$a_{1}$ & 0.02186 & 0.04333 \\
$a_{2}$ & -0.06330 & -0.1143 \\
$a_{3}$ & 0.00003149 & 0.0001108 \\
\hline
\end{tabular}

The parameters of the drift of a diffusion process are estimated using the nonparametric estimator of Ait-Sahalia $(1996 \mathrm{a}, \mathrm{b})$. The diffusion process is given by the following equation: $d r_{t}=\left(a_{0}+a_{1} r_{t}+\right.$ $\left.a_{2} r_{t}^{2}+a_{3} / r_{t}\right) d t+\sigma r_{t}^{0.5} d B_{t}$. The simulated data used in the estimation is drawn from a two-regime CIR model with the parameters from Panel A of Table 8. Column 2 contains the average estimates of the parameters over 5000 simulations, and Column 3 contains estimates from Aït-Sahalia (1996a, b) using the daily 7-day Eurodollar rate.

the above OLS procedure. In our nonparametric estimation of the stationary density, we choose the IID bandwidth similar to that used by Ait-Sahalia (1996b), $h=\hat{\sigma} n^{-0.2}$, where $\hat{\sigma}$ is the sample standard deviation of the data and $n$ is the sample size. The parameters used in our simulation are shown in panel A of Table 8 .

The estimates of $a_{0}$ through $a_{3}$ averaged over 5000 simulations are shown in column 2 of Table 9, along with the estimates by Aït-Sahalia (1996b) which use the Eurodollar rate. In Figure 3, the solid line represents the $\operatorname{drift} \mu\left(r_{t ;}, \theta\right)$ based on our estimates of $a_{0}$ through $a_{3}$, and the dashed line represents the drift based on Aït-Sahalia's estimates. The two drifts are very similar to each other. Both show significant mean-reversion for very low and very high interest rates, and zero drift in the middle range. Aït-Sahalia's estimates display stronger nonlinearity at both ends of the range of the data than ours do. One possible reason for this divergence is that the bias of the nonparametric method tends to make the nonlinearity stronger at the boundaries of the data. Since our 


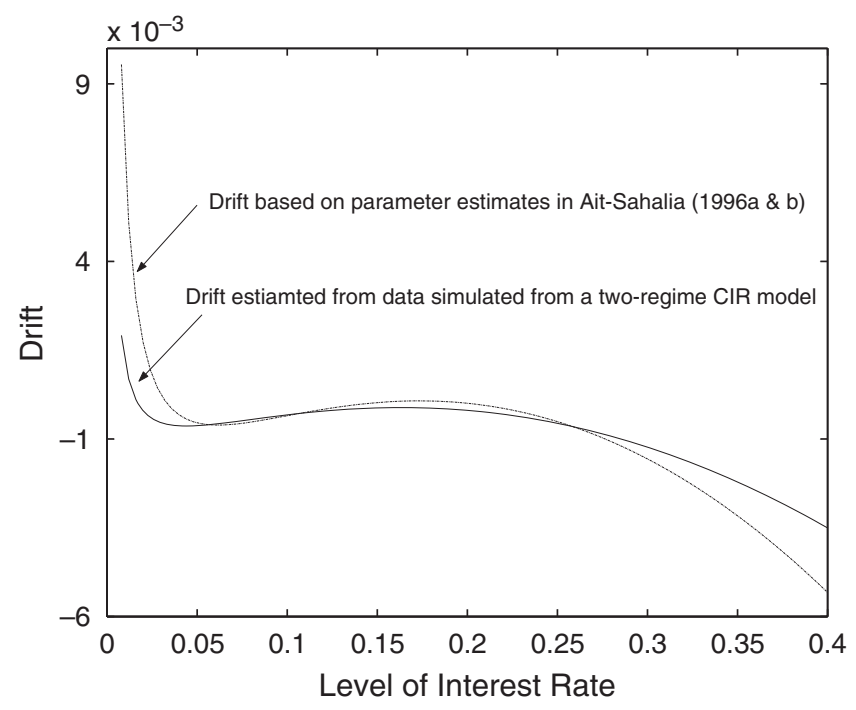

Figure 3 Drift Estimated Using Aït-Sahalia's Nonparametric Estimator.

The solid line represents the average drift estimated using Aït-Sahalia's nonparametric estimator based on data simulated from a two-regime CIR model over 5000 simulations. The parameters used to simulate the data are given in panel A of Table 8. The dashed line represents the drift estimated using Ait-Sahalia's method based on the 7-day Eurodollar rate.

estimates are averaged over 5000 simulations, that bias might have been reduced.

\section{B. Drift estimated using Stanton's method and local linear estimator}

We first introduce the nonparametric estimator of Stanton (1997), then apply it to the data simulated from the above regime-switching model. For robustness check, we repeat the analysis using the local polynomial estimator of Fan and Zhang (2003), which has superior finite sample performances than Stanton's method. For other advantages of the local polynomial approach, see Fan and Gijbels (1996).

Recall the time-homogeneous diffusion process in equation (1). In discrete time, we have

$$
E\left[r_{t+1}-r_{t} \mid r_{t}\right]=\mu\left(r_{t}\right) \Delta+o(\Delta)
$$

where $\Delta$ is the discrete time step in a sequence of observations of the process $r_{t}$, and $o(\Delta)$ denotes a function $\xi$ such that $\lim _{\Delta \rightarrow 0} \frac{\xi(\Delta)}{\Delta}=0$.

Let $\left\{r_{t}\right\}_{t=1}^{n}$ be a sample of size $n$ from the continuous-time process $r_{t}$, observed at the discrete interval $\Delta$. Furthermore, let $\left\{z_{i}\right\}_{i}^{N}$ be a set of $N$ points defining an equally spaced partition of a subset of the support of the stationary density. If the stationary density of $r_{t}$ is represented by $\pi(r)$, a kernel estimator takes 
the form

$$
\widehat{\pi}\left(Z_{i}\right) \equiv \frac{1}{n} \sum_{t=1}^{n} \frac{1}{h} K\left(\frac{r_{t}-z_{i}}{h}\right) \equiv \frac{1}{n} \sum_{t=1}^{n} K_{h}\left(r_{t}-z_{i}\right)
$$

for $i=1,2, \ldots, N$ where $K$ is a kernel function satisfying the condition

$$
\int_{-\infty}^{+\infty} K(y) d y=1
$$

The kernel function provides a method of weighting 'nearby' observations in order to construct a smoothed histogram. Stanton (1997) uses a Gaussian kernel:

$$
K(u)=\frac{1}{\sqrt{2 \pi}} \exp \left(-\frac{1}{2} u^{2}\right), \quad u \in(-\infty,+\infty) .
$$

The estimators in Stanton (1997) are based directly on the discretized version of the diffusion equation, i.e.

$$
\mu\left(r_{t}\right)=\frac{1}{\Delta} E\left[r_{t+1}-r_{t} \mid r_{t}\right]+\frac{o(\Delta)}{\Delta} .
$$

The essence of Stanton's approach is to apply the Nadaraya-Watson (NW) kernel regression estimator to construct nonparametric estimators of the conditional expectations in the above equation:

$$
\widehat{\mu}\left(z_{i}\right)=\frac{1}{\Delta} \frac{\sum_{t=1}^{n-1}\left(r_{t+1}-r_{t}\right) K_{h}\left(r_{t}-z_{i}\right)}{\sum_{t=1}^{n-1} K_{h}\left(r_{t}-z_{i}\right)} .
$$

While the NW estimator approximates the drift function by a constant in the neighborhood of $z_{i}$, the local polynomial approach approximates it by a polynomial. That is,

$$
\begin{aligned}
\mu\left(r_{t}\right) & \approx \mu\left(z_{i}\right)+\mu^{\prime}\left(z_{i}\right)\left(r_{t}-z_{i}\right)+\ldots+\frac{\mu^{(p)}\left(z_{i}\right)}{p !}\left(r_{t}-z_{i}\right)^{p} \\
& \left.=\sum_{k=0}^{p} \beta_{k}\left(z_{i}\right)\right) \times\left(r_{t}-z_{i}\right)^{k}
\end{aligned}
$$

where $\beta_{k}\left(z_{i}\right)=\mu^{(k)}\left(z_{i}\right) / k$ ! and $\mu^{(k)}\left(z_{i}\right)=\left.\frac{\partial \mu^{k}(z)}{\partial z^{k}}\right|_{z=z_{i}}$.

In our analysis, we estimate $\mu(\cdot)$ using a locally linear estimator by solving the following weighted least square problem for each $z_{i}$ :

$$
\min _{\beta_{0}\left(z_{i}\right), \beta_{1}\left(z_{i}\right)} \sum_{t=1}^{n-1}\left\{\left(r_{t+1}-r_{t}\right)-\hat{\beta}_{0}\left(z_{i}\right)-\hat{\beta}_{1}\left(z_{i}\right)\left(r_{t}-z_{i}\right)\right\}^{2} K_{h}\left(r_{t}-z_{i}\right) .
$$

From the above setup, it is obvious that the NW estimator is a special case of the local polynomial method with $p=0$.

To match the size of Stanton's sample, we simulate a time series with 7500 observations for 5000 times. For each series, we estimate the drift using the simulated data. We use the same bandwidth as that used by Stanton (1997), $h=4 \times \hat{\sigma} n^{-0.2}$, where $\hat{\sigma}$ is the sample standard deviation of the data and $n$ is the 


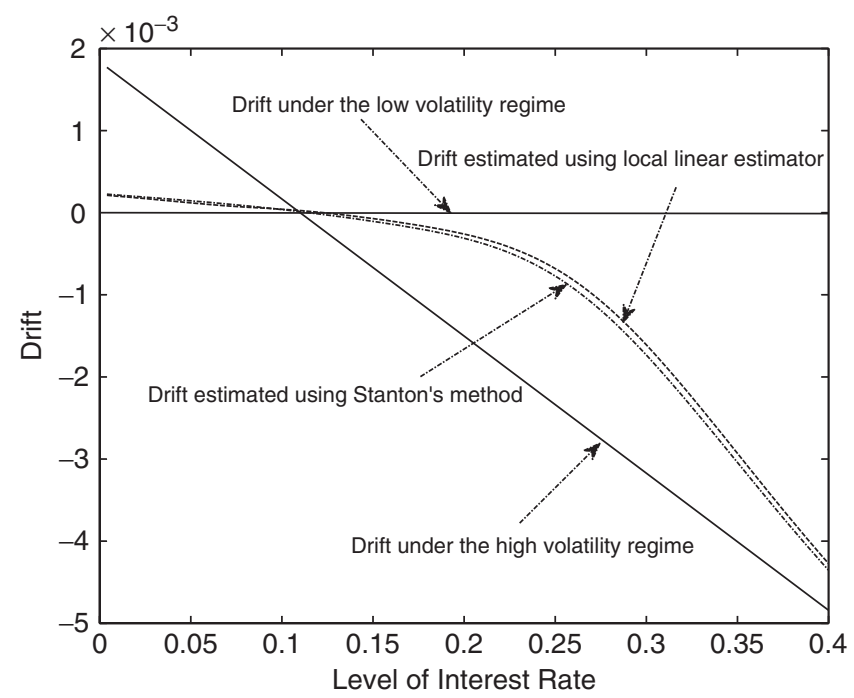

Figure 4 Drift Estimated Using Stanton's Method and Local Linear Estimator. The two dashed lines represent the average drift estimated using Stanton's method and local linear estimator based on data simulated from a two-regime CIR model over 5000 simulations. The two solid lines represent the drift in the two different regimes. The parameters used to simulate the data are given in panel B of Table 8 .

sample size. The parameters used in our simulation are mainly based on our estimates of the regime-switching model using Aït-Sahalia's data and are shown in panel B of Table $8 .^{8}$

Figure 4 plots the averaged estimate of the drift over the 5000 simulations. The two solid lines represent the drift of the short rate in two different regimes, while the two dashed lines represent the drift estimated using Stanton's estimator and the local linear estimator. From the graph, we can clearly see that if the data generated from our regime-switching model is treated as a singleregime model and estimated using either Stanton's method or the local linear estimator, nonlinear drift will appear exactly as found by Stanton who uses actual interest rate data in his analysis.

One difference between the drift in Figure 4 and that found by Stanton (1997) is that Stanton's drift becomes negative when the interest rate is about $15 \%$; our drift becomes negative at an interest rate of $20 \%$. The highest rate in

8 Notice that the parameters for the second regime are almost the same as those estimated from Ait-Sahalia's data. Since we simulate noncentral Chi-square distribution by taking the summation of independent IID standard normal random variables, we adjust $\alpha(1)$ in order to make the degree of freedom of the Chi-square distribution an integer. The parameters for the first regime are quite different from the estimated values. Since our focus is to estimate the speed of mean-reversion, $\beta(0)$ is chosen to be the same as that estimated from the data. However, we adjust $\alpha(0)$ and $\sigma(0)$ so that the degree of freedom of the Chi-square distribution is an integer, and the long-run mean $-\alpha(0) / \beta(0)$ is relatively low. 
Stanton's sample is $18 \%$, but it is $24 \%$ in Ait-Sahalia's sample. Since our parameters are calibrated to Aït-Sahalia's data, the differences between the two data sets could explain why the nonlinearity in the drift happens at a higher interest rate in our estimates.

Our analysis shows that although the nonparametric estimators of AitSahalia (1996b) and Stanton (1997) could yield nonlinear drift when the true drift is linear, the nonlinear drift could also be consistent with a data generating process that follows our two-regime model whose drift is truly nonlinear. Ang and Bekaert (1998) also discuss the implications of a regime-switching model for the drift of the short rate. They show that a regime-switching model with state-dependent transition probability can reproduce the shape of the drift estimated using nonparametric methods. Given the evidence, it seems that nonlinear drift is an important feature of the US short rate and should be taken seriously in theoretical models of term structure of interest rates.

\section{CONCLUSION}

In this paper, we estimate and test a Markov regime-switching model for the US short-term interest rate. We show that the short rate follows a regime-switching model with two different regimes: In one regime, the interest rate behaves like a random walk with low volatility, while in another it exhibits strong meanreversion and high volatility. This model has interesting implications for the dynamics of the short rate.

First, it shows that the sensitivity of interest rate volatility to the level of interest rate is not as high as commonly believed. This confirms the prediction of Campbell et al. (1997) that a two-regime CIR model could provide a good description of the time series behavior of the short rate over the past half century.

Second, our model is more flexible than the traditional ones in modeling the conditional volatility of the interest rate. Unlike the traditional models in which volatility solely depends on the level of interest rate, our model allows volatility to be low while interest rate is high, and volatility to be high while interest rate is low.

Finally, we show that the findings of nonlinear drift in Aitt-Sahalia (1996b) and Stanton (1997) are consistent with our regime-switching model in which the drift of the short rate is truly nonlinear. This suggests that nonlinear drift is indeed an important feature of the short rate process.

Haitao Li

Stephen M. Ross School of Business

University of Michigan

Ann Arbor

MI 48109

USA

htli@umich.edu 


\section{REFERENCES}

Ahn, D., and B. Gao (1999), 'A Parametric Nonlinear Model of Term Structure Dynamics', Review of Financial Studies, 12, 721-62.

Aït-Sahalia, Y. (1996a), 'Nonparametric Pricing of Interest Rate Derivative Securities', Econometrica, 64, 527-60.

Ait-Sahalia, Y. (1996b), 'Testing Continuous-Time Models of the Spot Interest Rate', Review of Financial Studies, 9, 385-426.

Andersen, T. G., and J. Lund (1997), 'Estimating Continuous Time Stochastic Volatility Models of the Short Term Interest Rate', Journal of Econometrics, 77, 343-78.

Andrews, D., and W. Ploberger (1994), 'Optimal Tests When a Nuisance Parameter is Present Only Under the Alternative', Econometrica, 62, 1383-414.

Ang, A., and G. Bekaert (1998), Regime Switches in Interest Rates, NBER Working Paper No. 6508.

Bansal, R., and H. Zhou (2002), 'Term Structure of Interest Rates with Regime Shifts', Journal of Finance, 57, 1997-2043.

Beaglehole, D.R., and M. Tenney (1992), 'A Nonlinear Equilibrium Model of the Term Structure of Interest Rates: Corrections and Additions', Journal of Financial Economics, 32, 345-54.

Bekaert, G., R. Hodrick, and D. Marshall (2001), "Peso Problem" Explanations for Term Structure Anomalies, Journal of Monetary Economics, 48, 241-70.

Bliss, R., and D. Smith (1998), The Elasticity of Interest Rate Volatility: Chan, Karolyi, Longstaff and Sanders Revisited, Journal of Risk, 1, 21-46.

Brennan, M., and E. Schwartz (1979), 'A Continuous-Time Approach to the Pricing of Bonds', Journal of Banking and Finance, 3, 133-55.

Brenner, R., R. Harjes, and K. Kroner (1996), 'Another Look at Alternative Models of Short-Term Interest Rate', Journal of Financial and Quantitative Analysis, 31, 85-107.

Brown, S., and P. Dybvig (1986), 'The Empirical Implications of the Cox, Ingersoll, Ross Theory of the Term Structure of Interest Rates', Journal of Finance, 41, 617-30.

Campbell, J., and A. Lo, and C. MacKinlay (1997), The Econometrics of Financial Markets. New Jersey: Princeton University Press.

Chan, K. C., G. A. Karolyi, F. A. Longstaff, and A. B. Sanders (1992), 'An Empirical Comparison of Alternative Models of the Short-Term Interest Rate', Journal of Finance, 47, 1209-27.

Chapman, D., J. Long, and N. Pearson (1999), 'Using Proxies for the Short Rate: When Are Three Months Like an Instant?', Review of Financial Studies, 12, 763-806.

Chapman, D., and N. Pearson (2000), 'Is the Short Rate Drift Actually Nonlinear?', Journal of Finance, 55, 355-88.

Conley, T. G., L. P. Hansen, E. G. J. Luttmer, and J. A. Scheinkman (1997), 'Short-Term Interest Rates as Subordinated Diffusions', Review of Financial Studies, 10, 525-78.

Constantinides, G. (1992), 'A Theory of the Nominal Term Structure of Interest Rates', Review of Financial Studies, 5, 531-52.

Courtadon, G. (1982), 'The Pricing of Options on Default Free Bonds', Journal of Financial and Quantitative Analysis, 17, 75-100.

Cox, J. C., J. E. Ingersoll, and S. A. Ross (1980), 'An Analysis of Variable rate Loan Contracts', Journal of Finance, 35, 389-403.

Cox, J. C., J. E. Ingersoll, and S. A. Ross (1985), 'A Theory of the Term Structure of Interest Rates', Econometrica, 53, 385-407.

Dai, Q., K. Singleton, and W. Yang (2007), 'Regime Shifts in a Dynamic Term Structure Model of U.S. Treasury Bond Yields', Review of Financial Studies, 20, 1669-706.

Davies, R. (1977), 'Hypothesis Testing When a Nuisance Parameter is Present Only Under the Alternatives', Biometrika, 64, 247-54.

Davies, R. (1987), 'Hypothesis Testing When a Nuisance Parameter is Present Only Under the Alternatives', Biometrika, 74, 33-43. 
Dothan, L. U. (1978), 'On the Term Structure of Interest Rates', Journal of Financial Economics, 6, 59-69.

Duffie, D., and R. Kan (1996), 'A Yield Factor Model of Interest Rates', Mathematical Finance, 6, 379-406.

Fan, J., and I. Gijbels (1996), Local Polynomial Modeling and its Applications. London: Chapman and Hall.

Fan, J., and C. Zhang (2003), 'A Reexamination of Diffusion Estimators With Applications to Financial Model Validation', Journal of American Statistical Association, 98, 118-34.

Garcia, R. (1998), 'Asymptotic Null Distribution of the Likelihood Ratio Test in Markov Switching Models', International Economic Review, 39, 763-84.

Gay, D. (1983), 'Algorithm 611, Subroutines for Unconstrained Minimization Using a Model/Trust-Region Approach', ACM Transactions on Mathematical Software, 9, 503-24.

Gray, S. (1996), 'Modeling the Conditional Distribution of Interest Rates as a RegimeSwitching Process', Journal of Financial Economics, 42, 27-62.

Hamilton, J. D. (1989), 'A New Approach to the Econometric Analysis of Nonstationary Time Series and the Business Cycle', Econometrica, 57, 357-84.

Hamilton, J. D. (1994), Time Series Analysis. Princeton, NJ: Princeton University Press.

Hansen, B. E. (1992), 'The Likelihood Ratio Test Under Nonstandard Conditions: Testing the Markov Switching model of GNP', Journal of Applied Econometrics, 7, 61-82.

Hansen, B. E. (1996), 'Inference When a Nuisance Parameter in Not Identified Under the Null Hypothesis', Econometrica, 64, 413-30.

Longstaff, F., and E. Schwartz (1992), 'Interest Rate Volatility and the Term Structure: A Two-Factor General Equilibrium Model', Journal of Finance, 47, 1259-82.

Marsh, T. A., and E. R. Rosenfeld (1983), 'Stochastic Processes for Interest Rates and Equilibrium Bond Prices', Journal of Finance, 38, 635-46.

Merton, R. (1973), 'Theory of Rational Option Pricing', Bell Journal of Economics and Management Science, 4, 141-83.

Pritsker, M. (1998), 'Nonparametric Density Estimation and Tests of Continuous Time Interest Rate Models', Review of Financial Studies, 11, 449-87.

Stanton, R. (1997), 'A Nonparametric Model of Term Structure Dynamics and the Market Price of Interest Rate Risk', Journal of Finance, 52, 1973-2002.

Vasicek, O. (1977), 'An Equilibrium Characterization of the Term Structure', Journal of Financial Economics, 5, 177-188.

\section{APPENDIX A}

Proof of Lemma 1. In what follows, we derive a representation of the score function. Observe that

$$
\begin{aligned}
\frac{\partial p\left(r_{n}, \ldots, r_{1}, s_{n}, \ldots, s_{1} ; \vartheta, \eta\right)}{\partial \vartheta_{i}} & \frac{\partial \log p\left(r_{n}, \ldots, r_{1}, s_{n}, \ldots, s_{1} ; \vartheta, \eta\right)}{\partial \vartheta_{i}} p\left(r_{n}, \ldots, r_{1}, s_{n}, \ldots, s_{1} ; \vartheta, \eta\right) \\
= & \\
=\sum_{t=1}^{n} \frac{\partial \log p\left(r_{t} \mid S_{t}, \Psi_{t-1} ; \vartheta, \eta\right)}{\partial \vartheta_{i}} & \quad \times p\left(r_{n}, \ldots, r_{1} ; \vartheta, \eta\right) p\left(S_{n}=s_{n}, \ldots, S_{1}=s_{1} \mid r_{n}, \ldots, r_{1} ; \vartheta, \eta\right) .
\end{aligned}
$$


Then, summing over $s_{t}=0,1$ for $t=1, \ldots, n$ and dividing by $p\left(r_{n}, \ldots, r_{1} ; \theta\right)$, we obtain

$$
\begin{aligned}
& \frac{\partial \log p\left(r_{n}, \ldots, r_{1} ; \vartheta, \eta\right)}{\partial \vartheta_{i}} \frac{1}{p\left(r_{n}, \ldots, r_{1} ; \theta\right)} \\
& =\sum_{t=1}^{n} \frac{\partial \log p\left(r_{t} \mid S_{t}, \Psi_{t-1}, \theta\right)}{\partial \vartheta_{i}} p\left(S_{t}=s_{t}, \ldots, S_{1}=s_{1} \mid r_{n}, \ldots, r_{1} ; \theta\right) \\
& =\sum_{s_{n}=0}^{1} \frac{\partial \log p\left(r_{n} \mid S_{t}, \Psi_{n-1}, \theta\right)}{\partial \vartheta_{i}} p\left(S_{n}=s_{n} \mid r_{n}, \ldots, r_{1} ; \theta\right)+\ldots \\
& \quad+\sum_{s_{1}=0}^{1} \frac{\partial \log p\left(r_{1} \mid S_{t}, \Psi_{0}, \theta\right)}{\partial \vartheta_{i}} p\left(S_{1}=s_{1} \mid r_{n}, \ldots, r_{1} ; \theta\right)
\end{aligned}
$$

since $p\left(r_{t} \mid S_{t}, \Psi_{t-1}, \theta\right)$ depends only on $s_{t}$. The conditional probabilities $p\left(S_{t}=\right.$ $\left.s_{t} \mid r_{n}, \ldots, r_{1}, \theta\right)$ are the so-called smoothed probabilities. This provides the explicit representation of the scores.

To simplify notation, we denote $\varepsilon_{t}=\frac{r_{t}-\left(\alpha_{1}+\beta_{1} r_{t-1}\right)-\left(\alpha_{2} S_{t}+\beta_{2} r_{t-1} S_{t}\right)}{\left(\sigma_{1}+\sigma_{2} S_{t}\right) r_{t-1}^{?}}$. Now we can derive the score functions with $\eta=(p, q)$ fixed. Observe that

$$
\begin{aligned}
\frac{\partial \log p\left(r_{t} \mid S_{t}, \Psi_{t-1}\right)}{\partial \alpha_{1}} & =\frac{\partial}{\partial \alpha_{1}}\left[-\frac{\varepsilon_{t}^{2}}{2}-\log \left[\left(\sigma_{1}+\sigma_{2} S_{t}\right) r_{t-1}^{\gamma}\right]\right] \\
& =-\frac{2 \varepsilon_{t}}{2} \frac{-1}{\left(\sigma_{1}+\sigma_{2} S_{t}\right) r_{t-1}^{\gamma}}=\frac{\varepsilon_{t}}{\left(\sigma_{1}+\sigma_{2} S_{t}\right) r_{t-1}^{\gamma}} \\
\frac{\partial \log p\left(r_{t} \mid S_{t}, \Psi_{t-1}\right)}{\partial \alpha_{2}} & =\frac{\partial}{\partial \alpha_{2}}\left[-\frac{\varepsilon_{t}^{2}}{2}-\log \left[\left(\sigma_{1}+\sigma_{2} S_{t}\right) r_{t-1}^{\gamma}\right]\right] \\
& =-\frac{2 \varepsilon_{t}}{2} \frac{\left[-S_{t}\right]}{\left(\sigma_{1}+\sigma_{2} S_{t}\right) r_{t-1}^{\gamma}}=\frac{\varepsilon_{t} S_{t}}{\left(\sigma_{1}+\sigma_{2} S_{t}\right) r_{t-1}^{\gamma}} \\
\frac{\partial \log p\left(r_{t} \mid S_{t}, \Psi_{t-1}\right)}{\partial \beta_{1}} & =\frac{\partial}{\partial \beta_{1}}\left[-\frac{\varepsilon_{t}^{2}}{2}-\log \left[\left(\sigma_{1}+\sigma_{2} S_{t}\right) r_{t-1}^{\gamma}\right]\right] \\
& =-\frac{2 \varepsilon_{t}}{2} \frac{-r_{t-1}}{\left(\sigma_{1}+\sigma_{2} S_{t}\right) r_{t-1}^{\gamma}}=\frac{\varepsilon_{t} r_{t-1}}{\left(\sigma_{1}+\sigma_{2} S_{t}\right) r_{t-1}^{\gamma}} \\
\frac{\partial \log p\left(r_{t} \mid S_{t}, \Psi_{t-1}\right)}{\partial \beta_{2}} & =\frac{\partial}{\partial \beta_{2}}\left[-\frac{\varepsilon_{t}^{2}}{2}-\log \left[\left(\sigma_{1}+\sigma_{2} S_{t}\right) r_{t-1}^{\gamma}\right]\right] \\
& =-\frac{2 \varepsilon_{t}}{2} \frac{\left[-r_{t-1} S_{t}\right]}{\left(\sigma_{1}+\sigma_{2} S_{t}\right) r_{t-1}^{\gamma}}=\frac{\varepsilon_{t} r_{t-1} S_{t}}{\left(\sigma_{1}+\sigma_{2} S_{t}\right) r_{t-1}^{\gamma}}
\end{aligned}
$$




$$
\begin{aligned}
\frac{\partial \log p\left(r_{t} \mid S_{t}, \Psi_{t-1}\right)}{\partial \sigma_{1}} & =\frac{\partial}{\partial \sigma_{1}}\left[-\frac{\varepsilon_{t}^{2}}{2}-\log \left[\left(\sigma_{1}+\sigma_{2} S_{t}\right) r_{t-1}^{\gamma}\right]\right] \\
& =-\frac{2 \varepsilon_{t}}{2} \frac{\left[-r_{t-1}^{\gamma}\right] \varepsilon_{t}}{\left(\sigma_{1}+\sigma_{2} S_{t}\right) r_{t-1}^{\gamma}}-\frac{r_{t-1}^{\gamma}}{\left(\sigma_{1}+\sigma_{2} S_{t}\right) r_{t-1}^{\gamma}} \\
& =\frac{1}{\left(\sigma_{1}+\sigma_{2} S_{t}\right)}\left[\varepsilon_{t}^{2}-1\right] . \\
\frac{\partial \log p\left(r_{t} \mid S_{t}, \Psi_{t-1}\right)}{\partial \sigma_{2}} & =\frac{\partial}{\partial \sigma_{2}}\left[-\frac{\varepsilon_{t}^{2}}{2}-\log \left[\left(\sigma_{1}+\sigma_{2} S_{t}\right) r_{t-1}^{\gamma}\right]\right] \\
& =-\frac{2 \varepsilon_{t}}{2} \frac{\varepsilon_{t} S_{t} r_{t-1}^{\gamma}}{\left(\sigma_{1}+\sigma_{2} S_{t}\right) r_{t-1}^{\gamma}}-\frac{S_{t} r_{t}^{\gamma}}{\left(\sigma_{1}+\sigma_{2} S_{t}\right) r_{t-1}^{\gamma}} \\
& =\frac{S_{t}}{\left(\sigma_{1}+\sigma_{2} S_{t}\right)}\left[\varepsilon_{t}^{2}-1\right] .
\end{aligned}
$$

Using

$$
d r_{t}^{\gamma} / d \gamma=d \exp \left(\gamma \ln r_{t-1}\right) / d \gamma=\exp \left(\gamma \ln r_{t-1}\right) \ln r_{t-1}=r_{t-1}^{\gamma} \ln r_{t-1}
$$

we obtain

$$
\begin{aligned}
\frac{\partial \log p\left(r_{t} \mid S_{t}, \Psi_{t-1}\right)}{\partial \gamma}= & \frac{\partial}{\partial \gamma}\left[-\frac{\varepsilon_{t}^{2}}{2}-\log \left[\left(\sigma_{1}+\sigma_{2} S_{t}\right) r_{t-1}^{\gamma}\right]\right] \\
= & -\frac{2 \varepsilon_{t}}{2} \frac{\left[-\varepsilon_{t}\left(\sigma_{1}+\sigma_{2} S_{t}\right) r_{t-1}^{\gamma} \ln r_{t-1}\right]}{\left(\sigma_{1}+\sigma_{2} S_{t}\right) r_{t-1}^{\gamma}} \\
& -\frac{\left(\sigma_{1}+\sigma_{2} S_{t}\right) r_{t-1}^{\gamma} \ln r_{t-1}}{\left(\sigma_{1}+\sigma_{2} S_{t}\right) r_{t-1}^{\gamma}} \\
= & \varepsilon_{t}^{2} \ln r_{t-1}-\ln r_{t-1}=\ln r_{t-1}\left[\varepsilon_{t}^{2}-1\right]
\end{aligned}
$$

Evaluating these scores at the true distribution represented by $\theta_{0}=\left(\xi_{0}, \zeta_{0}, \eta\right)$ with $\xi_{0}=\left(\alpha_{0}, \beta_{0}, \sigma_{0}, \gamma_{0}\right), \xi_{0}=(0,0,0)$, we get

$$
\begin{gathered}
\left.\frac{\partial \log p\left(r_{t} \mid S_{t}, \Psi_{t-1}\right)}{\partial \alpha_{1}}\right|_{\theta_{0}}=\frac{\varepsilon_{t}}{\sigma_{0} r_{t-1}^{\gamma}},\left.\frac{\partial \log p\left(r_{t} \mid S_{t}, \Psi_{t-1}\right)}{\partial \alpha_{2}}\right|_{\theta_{0}}=\frac{\varepsilon_{t} S_{t}}{\sigma_{0} r_{t-1}^{\gamma}} \\
\left.\frac{\partial \log p\left(r_{t} \mid S_{t}, \Psi_{t-1}\right)}{\partial \beta_{1}}\right|_{\theta_{0}}=\frac{\varepsilon_{t}}{\sigma_{0} r_{t-1}^{\gamma-1}},\left.\frac{\partial \log p\left(r_{t} \mid S_{t}, \Psi_{t-1}\right)}{\partial \beta_{2}}\right|_{\theta_{0}}=\frac{\varepsilon_{t} S_{t}}{\sigma_{0} r_{t-1}^{\gamma-1}} \\
\left.\frac{\partial \log p\left(r_{t} \mid S_{t}, \Psi_{t-1}\right)}{\partial \sigma_{1}}\right|_{\theta_{0}}=\frac{1}{\sigma_{0}}\left[\varepsilon_{t}^{2}-1\right],\left.\frac{\partial \log p\left(r_{t} \mid S_{t}, \quad \Psi_{t-1}\right)}{\partial \sigma_{2}}\right|_{\theta_{0}}=\frac{S_{t}}{\sigma_{0}}\left[\varepsilon_{t}^{2}-1\right] \\
\left.\frac{\partial \log p\left(r_{t} \mid S_{t}, \quad \Psi_{t-1}\right)}{\partial \gamma}\right|_{\theta_{0}}=\ln r_{t-1}\left[\varepsilon_{t}^{2}-1\right] .
\end{gathered}
$$

It follows that the score functions are given by those in the Lemma. 
Proof of Lemma 2. For $\eta=(p, q)$ and $\eta^{\prime}=\left(p^{\prime}, q^{\prime}\right), \Lambda\left(\eta, \eta^{\prime}\right)$ is a $7 \times 7$ matrix. Here we illustrate how each element can be calculated:

$$
\begin{aligned}
n E Z_{\alpha_{1}} Z_{\alpha_{1}} & =\frac{1}{n} E\left(\sum_{k=1}^{n} \frac{\varepsilon_{k}}{\sigma_{0} r_{k-1}^{\gamma}}\right)^{2}=\frac{1}{n} E \sum_{k=1}^{n} \sum_{l=1}^{n} \frac{\varepsilon_{k} \varepsilon_{l}}{\sigma_{0}^{2} r_{k-1}^{\gamma} r_{l-1}^{\gamma}} \\
& =\frac{1}{n} \sum_{k=1}^{T} E \frac{\varepsilon_{k}^{2}}{\sigma_{0}^{2} r_{k-1}^{2 \gamma}}=\frac{1}{\sigma_{0}^{2}} \frac{1}{n} \sum_{k=1}^{n} E r_{k-1}^{-2 \gamma}=\frac{1}{\sigma_{0}^{2}} E r_{1}^{-2 \gamma}
\end{aligned}
$$

Similarly,

$$
\begin{aligned}
& n E Z_{\beta_{1}} Z_{\beta_{1}}=\frac{1}{n} E\left(\sum_{k=1}^{n} \frac{\varepsilon_{k}}{\sigma_{0} r_{k-1}^{\gamma-1}}\right)^{2}=\frac{1}{\sigma_{0}^{2}} \frac{1}{n} \sum_{k=1}^{n} E r_{k-1}^{-2 \gamma-2}=\frac{1}{\sigma_{0}^{2}} E r_{1}^{-2 \gamma-2} \\
& n E Z_{\sigma_{1}} Z_{\sigma_{1}}=\frac{1}{n} E\left(\sum_{t=1}^{n} \frac{1}{\sigma_{0}}\left[\varepsilon_{t}^{2}-1\right]\right)^{2}=\frac{1}{\sigma_{0}^{2}} \frac{1}{n} \sum_{t=1}^{n} E\left[\varepsilon_{t}^{2}-1\right]^{2}=\frac{2}{\sigma_{0}^{2}} \\
& n E Z_{\gamma} Z_{\gamma}=\frac{1}{n} E\left(\sum_{t=1}^{n} \ln r_{t-1}\left[\varepsilon_{t}^{2}-1\right]\right)^{2} \\
& =\frac{1}{n} E \sum_{t=1}^{n} E\left[\ln r_{t-1}\right]^{2}\left[\varepsilon_{t}^{2}-1\right]^{2}=\frac{2}{\sigma_{0}^{2}} E\left[\ln r_{1}\right]^{2} \\
& n E Z_{\alpha_{1}} Z_{\beta_{1}}=E \sum_{k=1}^{n} \sum_{l=1}^{n} \frac{\varepsilon_{k} \varepsilon_{l}}{\sigma_{0}^{2} r_{k-1}^{\gamma} r_{l-1}^{\gamma-1}}=\frac{1}{\sigma_{0}^{2}} E r_{1}^{-2 \gamma+1} \\
& n E Z_{\alpha_{1}} Z_{\sigma_{1}}=E \sum_{k=1}^{n} \sum_{l=1}^{n} \frac{\varepsilon_{k}}{\sigma_{0}^{2} r_{k-1}^{\gamma}}\left[\varepsilon_{l}^{2}-1\right] \\
& =E \sum_{k=1}^{n} \frac{\varepsilon_{k}}{\sigma_{0}^{2} r_{k-1}^{\gamma}}\left[\varepsilon_{k}^{2}-1\right]=0 \\
& n E Z_{\alpha_{1}} Z_{\gamma}=E \sum_{k=1}^{n} \sum_{l=1}^{n} \frac{\varepsilon_{k}}{\sigma_{0}^{2} r_{k-1}^{\gamma}}\left[\ln r_{k-1}\right]\left[\varepsilon_{l}^{2}-1\right]=0 \\
& n E Z_{\beta_{1}} Z_{\sigma_{1}}=E \sum_{k=1}^{n} \sum_{l=1}^{n} \frac{\varepsilon_{k}}{\sigma_{0} r_{k-1}^{\gamma-1}}\left[\varepsilon_{l}^{2}-1\right]=0 \\
& n E Z_{\beta_{1}} Z_{\gamma}=0 \\
& n E Z_{\sigma_{1}} Z_{\gamma}=E \sum_{k=1}^{n} \sum_{l=1}^{n} \frac{1}{\sigma_{0}}\left[\varepsilon_{k}^{2}-1\right]\left[\varepsilon_{l}^{2}-1\right] \ln r_{l-1} \\
& =\frac{1}{\sigma_{0}} E \sum_{k=1}^{n}\left[\varepsilon_{k}^{2}-1\right]^{2} \ln r_{k-1}=\frac{2}{\sigma_{0}} E \ln r_{1} \text {. }
\end{aligned}
$$


To derive other covariances, note that $p_{t}$ is the conditional probability of the state variable conditioning on the data under the null. Hence the conditioning $\sigma$-field is generated by the shocks $\varepsilon_{t}$ only. It follows that

$$
\begin{aligned}
n E Z_{\alpha_{1}} Z_{\alpha_{2}} & =E\left[\sum_{t=1}^{n} \frac{\varepsilon_{t}}{\sigma_{0} r_{t-1}^{\gamma}}\right]\left[\sum_{l=1}^{n} \sum_{S_{l}} \frac{\varepsilon_{l} S_{l}}{\sigma_{0} r_{l-1}^{\gamma}} p_{l}\right]=E\left[\sum_{t=1}^{n} \sum_{l=1}^{n} \sum_{S_{l}} \frac{\varepsilon_{t} \varepsilon_{l} S_{l}}{\sigma_{0}^{2} r_{t-1}^{\gamma} r_{l-1}^{\gamma}} p_{l}\right] \\
& =E \sum_{t=1}^{n} \sum_{l=1}^{n} \frac{\varepsilon_{t} \varepsilon_{l}}{\sigma_{0}^{2} r_{t-1}^{\gamma} r_{l-1}^{\gamma}} E\left[S_{l} \mid y_{1}, \ldots, y_{n}\right] \\
& =E \sum_{t=1}^{n} \sum_{l=1}^{n} \frac{\varepsilon_{t} \varepsilon_{l}}{\sigma_{0}^{2} r_{t-1}^{\gamma} r_{l-1}^{\gamma}} E S_{l} \text { (since } S_{l} \text { are independent of } y_{1}, \ldots y_{n} \text { under the null) } \\
& =E \sum_{t=1}^{n} \sum_{l=1}^{n} \frac{\varepsilon_{t} \varepsilon_{l}}{\sigma_{0}^{2} r_{t-1}^{\gamma} r_{l-1}^{\gamma}} \pi^{\prime}=\frac{\pi^{\prime}}{\sigma_{0}^{2}} E r_{1}^{-2 \gamma}
\end{aligned}
$$

where $\pi^{\prime}=\left(1-q^{\prime}\right) /\left(2-q^{\prime}-p^{\prime}\right)$.

Similarly,

$$
\begin{aligned}
& n E Z_{\alpha_{1}} Z_{\beta_{2}}=E\left[\sum_{t=1}^{n} \frac{\varepsilon_{t}}{\sigma_{0} r_{t-1}^{\gamma}}\right]\left[\sum_{l=1}^{n} \sum_{S_{l}} \frac{\varepsilon_{l} S_{l}}{\sigma_{0} r_{l-1}^{\gamma-1}} p_{l}\right]=\frac{\pi^{\prime}}{\sigma_{0}^{2}} E r_{1}^{-2 \gamma+1} \\
& n E Z_{\alpha_{1}} Z_{\sigma_{2}}=E\left[\sum_{t=1}^{n} \frac{\varepsilon_{t}}{\sigma_{0} r_{t-1}^{\gamma}}\right]\left[\sum_{l=1}^{n} \sum_{S_{l}} \frac{S_{l}}{\sigma_{0}}\left[\varepsilon_{l}^{2}-1\right] p_{l}\right]=0 \\
& n E Z_{\beta_{1}} Z_{\alpha_{2}}=E \sum_{t=1}^{n} \frac{\varepsilon_{t}}{\sigma_{0} r_{t-1}^{\gamma-1}} \sum_{l=1}^{n} \sum_{S_{l}} \frac{\varepsilon_{l} S_{l}}{\sigma_{0} r_{l-1}^{\gamma}} p_{l}=\frac{\pi^{\prime}}{\sigma_{0}^{2}} E r_{1}^{-2 \gamma+1} \\
& n E Z_{\beta_{1}} Z_{\beta_{2}}=E \sum_{t=1}^{n} \frac{\varepsilon_{t}}{\sigma_{0} r_{t-1}^{\gamma-1}}\left[\sum_{l=1}^{n} \sum_{S_{l}} \frac{\varepsilon_{l} S_{l}}{\sigma_{0} r_{l-1}^{\gamma}} p_{l}\right]=\frac{\pi^{\prime}}{\sigma_{0}^{2}} E r_{1}^{-2 \gamma} \\
& n E Z_{\beta_{1}} Z_{\sigma_{2}}=E \sum_{t=1}^{n} \frac{\varepsilon_{t}}{\sigma_{0} r_{t-1}^{\gamma-1}}\left[\sum_{l=1}^{n} \sum_{S_{l}} \frac{S_{l}}{\sigma_{0}}\left[\varepsilon_{l}^{2}-1\right] p_{l}\right]=0 \\
& n E Z_{\sigma_{1}} Z_{\alpha_{2}}=E \sum_{t=1}^{n} \frac{1}{\sigma_{0}}\left[\varepsilon_{t}^{2}-1\right] \sum_{l=1}^{n} \sum_{S_{l}} \frac{\varepsilon_{l} S_{l}}{\sigma_{0} r_{l-1}^{\gamma}} p_{l}=0 \\
& n E Z_{\sigma_{1}} Z_{\beta_{2}}=E \sum_{t=1}^{n} \frac{1}{\sigma_{0}}\left[\varepsilon_{t}^{2}-1\right]\left[\sum_{l=1}^{n} \sum_{S_{l}} \frac{\varepsilon_{l} S_{l}}{\sigma_{0} r_{l-1}^{\gamma}} p_{l}\right]=0 \\
& n E Z_{\sigma_{1}} Z_{\sigma_{2}}=E \sum_{t=1}^{n} \frac{1}{\sigma_{0}}\left[\varepsilon_{t}^{2}-1\right]\left[\sum_{l=1}^{n} \sum_{S_{l}} \frac{S_{l}}{\sigma_{0}}\left[\varepsilon_{l}^{2}-1\right] p_{l}\right]=\frac{2 \pi^{\prime}}{\sigma_{0}^{2}} \\
& n E Z_{\gamma} Z_{\alpha_{2}}=E \sum_{t=1}^{n} \ln r_{t-1}\left[\varepsilon_{t}^{2}-1\right] \sum_{l=1}^{n} \sum_{S_{l}} \frac{\varepsilon_{l} S_{l}}{\sigma_{0} r_{l-1}^{\gamma}} p_{l}=0
\end{aligned}
$$




$$
\begin{aligned}
& n E Z_{\gamma} Z_{\beta_{2}}=E \sum_{t=1}^{n} \ln r_{t-1}\left[\varepsilon_{t}^{2}-1\right]\left[\sum_{l=1}^{n} \sum_{S_{l}} \frac{\varepsilon_{l} S_{l}}{\sigma_{0} r_{l-1}^{\gamma}} p_{l}\right]=0 \\
& n E Z_{\gamma} Z_{\sigma_{2}}=E \sum_{t=1}^{n} \ln r_{t-1}\left[\varepsilon_{t}^{2}-1\right]\left[\sum_{l=1}^{n} \sum_{S_{l}} \frac{S_{l}}{\sigma_{0}}\left[\varepsilon_{l}^{2}-1\right] p_{l}\right]=\frac{2 \pi^{\prime}}{\sigma_{0}^{2}} E \ln r_{1} \\
& n E Z_{\alpha_{2}}(\eta) Z_{\alpha_{2}}\left(\eta^{\prime}\right)=E\left[\sum_{t=1}^{n} \sum_{S_{t}} \frac{\varepsilon_{t} S_{t}}{\sigma_{0} r_{t-1}^{\gamma}} p_{t}\right]\left[\sum_{l=1}^{n} \sum_{S_{l}} \frac{\varepsilon_{l} S_{l}}{\sigma_{0} r_{l-1}^{\gamma}} p_{l}\right] \\
& =E\left[\sum_{t=1}^{n} \sum_{l=1}^{n} \sum_{S_{t}} \sum_{S_{l}} \frac{\varepsilon_{t} \varepsilon_{l} S_{t} S_{l}}{\sigma_{0}^{2} r_{t-1}^{\gamma} r_{l-1}^{\gamma}} p_{t} p_{l}\right]=E\left[\sum_{t=1}^{n} \sum_{l=1}^{n} \frac{\varepsilon_{t} \varepsilon_{l}}{\sigma_{0}^{2} r_{t-1}^{\gamma} r_{l-1}^{\gamma}} E S_{t} S_{l}\right] \\
& =E\left[\sum_{t=1}^{n} \sum_{l=1}^{n} \frac{\varepsilon_{t} \varepsilon_{l}}{\sigma_{0}^{2} r_{t-1}^{\gamma} r_{l-1}^{\gamma}}\right] \min \left(\pi, \pi^{\prime}\right)=\frac{1}{\sigma_{0}^{2}} E r_{1}^{-2 \gamma} \min \left(\pi, \pi^{\prime}\right) \\
& n E Z_{\beta_{2}} Z_{\beta_{2}}=E\left[\sum_{t=1}^{n} \sum_{S_{t}} \frac{\varepsilon_{t} S_{t}}{\sigma_{0} r_{t-1}^{\gamma-1}} p_{t}\right]\left[\sum_{l=1}^{n} \sum_{S_{l}} \frac{\varepsilon_{l} S_{l}}{\sigma_{0} r_{l-1}^{\gamma-1}} p_{l}\right] \\
& =\frac{1}{\sigma_{0}^{2}} E r_{1}^{-2 \gamma+2} \min \left(\pi, \pi^{\prime}\right) \\
& n E Z_{\sigma_{2}} Z_{\sigma_{2}}=E\left[\sum_{t=1}^{n} \sum_{S_{t}} \frac{S_{t}}{\sigma_{0}}\left[\varepsilon_{t}^{2}-1\right] p_{t}\right]\left[\sum_{s=1}^{n} \sum_{S_{s}} \frac{S_{s}}{\sigma_{0}}\left[\varepsilon_{s}^{2}-1\right] p_{s}\right] \\
& =E\left[\sum_{t=1}^{n} \sum_{s=1}^{n} \frac{\left[\varepsilon_{t}^{2}-1\right]\left[\varepsilon_{s}^{2}-1\right]}{\sigma_{0}^{2}} E S_{t} S_{s}\right]=\frac{2}{\sigma_{0}^{2}} \min \left(\pi, \pi^{\prime}\right) \\
& n E Z_{\alpha_{2}} Z_{\beta_{2}}=E\left[\sum_{t=1}^{n} \sum_{S_{t}} \frac{\varepsilon_{t} S_{t}}{\sigma_{0} r_{t-1}^{\gamma}} p_{t}\right]\left[\sum_{l=1}^{n} \sum_{S_{l}} \frac{\varepsilon_{l} S_{l}}{\sigma_{0} r_{l-1}^{\gamma-1}} p_{l}\right] \\
& =\frac{1}{\sigma_{0}^{2}} \operatorname{Er}_{1}^{-2 \gamma+1} \min \left(\pi, \pi^{\prime}\right) \\
& n E Z_{\alpha_{2}} Z_{\sigma_{2}}=E\left[\sum_{t=1}^{n} \sum_{S_{t}} \frac{\varepsilon_{t} S_{t}}{\sigma_{0} r_{t-1}^{\gamma}} p_{t}\right]\left[\sum_{l=1}^{n} \sum_{S_{l}} \frac{S_{l}}{\sigma_{0}}\left[\varepsilon_{l}^{2}-1\right] p_{l}\right]=0 \\
& n E Z_{\beta_{2}} Z_{\sigma_{2}}=E\left[\sum_{t=1}^{n} \sum_{S_{t}} \frac{\varepsilon_{t} S_{t}}{\sigma_{0} r_{t-1}^{\gamma-1}} p_{t}\right]\left[\sum_{l=1}^{n} \sum_{S_{l}} \frac{S_{l}}{\sigma_{0}}\left[\varepsilon_{l}^{2}-1\right] p_{l}\right]=0
\end{aligned}
$$

This yields the desired results. 